\title{
Human Galectin-9 Is a Potent Mediator of HIV Transcription and Reactivation
}

\author{
Mohamed Abdel-Mohsen ${ }^{1,2}$, Leonard Chavez ${ }^{1}$, Ravi Tandon ${ }^{3}$, Glen M. Chew ${ }^{4}$, \\ Xutao Deng ${ }^{1}$, Ali Danesh ${ }^{1,2}$, Sheila Keating ${ }^{1}$, Marion Lanteri ${ }^{1}$, Michael L. Samuels ${ }^{5}$, \\ Rebecca Hoh ${ }^{2}$, Jonah B. Sacha ${ }^{6,7}$, Philip J. Norris ${ }^{1,2}$, Toshiro Niki ${ }^{8,9}$, Cecilia M. Shikuma ${ }^{4}$, \\ Mitsuomi Hirashima ${ }^{8,9}$, Steven G. Deeks ${ }^{2}$, Lishomwa C. Ndhlovu ${ }^{4 \ddagger}$, Satish K. Pillai ${ }^{1,2 \ddagger *}$ \\ 1 Blood Systems Research Institute, San Francisco, California, United States of America, 2 University of \\ California, San Francisco, California, United States of America, 3 School of Biotechnology, Jawaharlal Nehru \\ University, New Delhi, India, 4 Hawaii Center for AIDS, John A. Burns School of Medicine, University of \\ Hawaii, Honolulu, Hawaii, United States of America, 5 RainDance Technologies, Inc., Billerica, \\ Massachusetts, United States of America, 6 Vaccine \& Gene Therapy Institute, Oregon Health \& Science \\ University, Portland, Oregon, United States of America, 7 Oregon National Primate Research Center, \\ Oregon Health \& Science University, Portland, Oregon, United States of America, 8 GalPharma Co., Ltd., \\ Takamatsu-shi, Kagawa, Japan, 9 Department of Immunology and Immunopathology, Kagawa University, \\ Kagawa, Japan
}

¥ These authors are joint senior authors on this work.

* satish.pillai@ucsf.edu

\section{Abstract}

Identifying host immune determinants governing HIV transcription, latency and infectivity in vivo is critical to developing an HIV cure. Based on our recent finding that the host factor p21 regulates HIV transcription during antiretroviral therapy (ART), and published data demonstrating that the human carbohydrate-binding immunomodulatory protein galectin-9 regulates p21, we hypothesized that galectin-9 modulates HIV transcription. We report that the administration of a recombinant, stable form of galectin- 9 ( $\mathrm{rGal}-9$ ) potently reverses HIV latency in vitro in the J-Lat HIV latency model. Furthermore, rGal-9 reverses HIV latency ex vivo in primary CD4+ T cells from HIV-infected, ART-suppressed individuals $(p=0.002)$, more potently than vorinostat $(p=0.02)$. rGal-9 co-administration with the latency reversal agent "JQ1", a bromodomain inhibitor, exhibits synergistic activity $(p<0.05)$. rGal-9 signals through $\mathrm{N}$-linked oligosaccharides and $\mathrm{O}$-linked hexasaccharides on the $\mathrm{T}$ cell surface, modulating the gene expression levels of key transcription initiation, promoter proximalpausing, and chromatin remodeling factors that regulate HIV latency. Beyond latent viral reactivation, rGal-9 induces robust expression of the host antiviral deaminase APOBEC3G in vitro and ex vivo (FDR<0.006) and significantly reduces infectivity of progeny virus, decreasing the probability that the HIV reservoir will be replenished when latency is reversed therapeutically. Lastly, endogenous levels of soluble galectin- 9 in the plasma of 72 HIV-infected ART-suppressed individuals were associated with levels of HIV RNA in CD4+ T cells $(p<0.02)$ and with the quantity and binding avidity of circulating anti-HIV antibodies $(p<0.009)$, suggesting a role of galectin- 9 in regulating HIV transcription and viral production in vivo during therapy. Our data suggest that galectin- 9 and the host glycosylation machinery should be explored as foundations for novel HIV cure strategies. 
Al027757 (LCN). The funders had no role in study design, data collection and analysis, decision to publish, or preparation of the manuscript.

Competing Interests: I have read the journal's policy and the authors of this manuscript have the following competing interests: MLS is currently employed by RainDance Technologies, Inc., which provided support for some of the droplet digital PCR assays performed in this study to measure HIV and host gene expression. TN and $\mathrm{MH}$ are employed by GalPharma Co., Ltd., which designed and provided the recombinant, stable form galectin- 9 used in our study, and supported experiments to evaluate the safety and tolerability of recombinant galactic-9 in the Lewis rat model. These relationships do not alter our adherence to all PLOS Pathogens policies on sharing data and materials.

\section{Author Summary}

While antiretroviral therapy (ART) has significantly decreased the morbidity and mortality associated with HIV infection, a cure is not achieved due to the persistence of HIV latently-infected cells during treatment. Identifying the principal host immune determinants governing HIV transcription, latency, and infectivity in vivo will be a critical step in developing an effective curative strategy for HIV infection. In this study, we demonstrate that the human immunomodulatory carbohydrate-binding protein galectin- 9 is a determinant of HIV latency in HIV-infected individuals on suppressive ART. Administration of galectin-9 potently reactivates latent $\mathrm{HIV}$ in $\mathrm{CD} 4+\mathrm{T}$ cells ex vivo, by signaling through specific glycans on the cell surface to modulate the gene expression levels of key host factors that regulate HIV transcription. Furthermore, galectin-9 induces the host APOBEC3 proteins which lethally mutagenize the HIV genome, attenuating viral infectivity. Our findings reveal a novel biological function of human galectin-9, and demonstrate that host glycans on the surface of infected cells mediate signals that define the transcriptional state and infectivity of HIV. Our findings suggest that galectin-9 and the host glycosylation machinery may be exploited to eradicate the latent HIV reservoir.

\section{Introduction}

Antiretroviral therapy (ART) has demonstrated efficacy and durability in suppressing HIV replication in infected individuals. However, ART does not achieve viral eradication due to the persistence of latently infected long-lived cells [1,2]. Several recent studies demonstrating continued morbidity during suppressive ART have created profound interest in developing a cure for HIV infection. The elimination of the latent reservoir is critical to achieving HIV eradication, as demonstrated by resurgence of virus post ART-cessation [3,4]. Alternatively, a "functional cure" involving control of virus to undetectable levels in the absence of complete eradication may be established to minimize ART-associated morbidity and enable ART-independent suppression of HIV to clinically undetectable levels, as demonstrated in recent clinical studies [5]. Identifying host determinants governing HIV transcription, latency, and infectivity in vivo will be a critical step in developing both of these curative modalities for HIV infection.

The "shock and kill" strategy is currently one of the most widely discussed approaches to eliminate the viral reservoir [6]. In this approach, drugs are administered to reverse HIV latency and induce viral production, ultimately resulting in the death of infected cells by direct viral cytopathic effects or immune-mediated clearance. Latency reversing agents (LRAs) are administered during suppressive ART, thereby preventing reactivated virus from replenishing the reservoir through infection of new cells. Clinical trials involving LRAs such as romidepsin, vorinostat, disulfiram, and panobinostat have failed to demonstrate significant reduction in reservoir size, although transient elevation in plasma viral RNA has been observed [7-13]. Accordingly, ex vivo experiments have revealed that the majority of existing LRAs exert weak effects on HIV transcription and reactivation [14]. The future success of shock and kill will depend on our capacity to design or identify highly efficacious LRAs and/or adjuvant therapies to boost the reactivation potential of existing LRAs.

Based on our recent finding that the p21 (CDKN1A) host restriction factor and cell cycle regulator $[15,16]$ modulates HIV transcription in ART-suppressed HIV-infected individuals [17], and reports suggesting that the human lectin galectin-9 (Gal-9) regulates p21 expression [18-20], we pursued the hypothesis that Gal-9 modulates HIV transcription and latency. The galectin family of animal lectins consists of a group of glycan-binding proteins characterized 
by conserved carbohydrate recognition domains (CRDs), defined by shared consensus amino acid sequences which confer specific binding to $\beta$-galactoside-containing glycoconjugate proteins [21]. Galectins are ubiquitously expressed throughout the animal kingdom, from lower organisms, such as nematodes and sponges, to higher mammalian species, including humans [22]. Fifteen members of the mammalian galectin family have been identified to date [23]. Gal9 has been recently recognized to play an important role in several diseases including HIV infection through regulation of both adaptive and innate defense mechanisms [24-26]. Recombinant Gal-9 (rGal-9) has been used successfully and safely as a therapy in a number of mouse disease models including graft versus host disease [27], rheumatoid arthritis [28], asthma [29], leukemia [30], and colon cancer [31].

\section{Results}

\section{rGal-9 potently reverses HIV latency in vitro}

We recently demonstrated that the expression levels of particular cell-intrinsic immune factors are associated with levels of cell-associated HIV RNA in the setting of ART [17]. Based on reports demonstrating that Gal-9 regulates the expression of these key cell-intrinsic immune mechanisms [18-20], we hypothesized that manipulation of Gal-9 may induce transcription of latent HIV. We initially assessed the ability of a biologically active and stable form of rGal-9 $[32,33]$ to modulate HIV transcription and reactivate latent HIV in the established "J-Lat" model of HIV latency. J-Lat cells harbor a latent, transcriptionally competent HIV provirus that encodes green fluorescent protein (GFP) as an indicator of reactivation [34,35]. We performed dose-response experiments by stimulating J-Lat 5A8, 6.3 and 11.1 clones [36] with rGal-9 for 24 hours. Our data revealed that rGal-9 reactivated latently-infected J-Lat cells in a dose-dependent manner (up to $17.9 \%$ in the $5 \mathrm{~A} 8$ clone, up to $6.96 \%$ in the 6.3 clone, and up to $40.1 \%$ in the 11.1 clone), and with potency greater than that seen with $\alpha \mathrm{CD} 3 / \alpha \mathrm{CD} 28$ stimulation (Fig 1A-1C). When we assessed the ability of other galectins (galectins-1, 3, 4, 7, 8) to reverse HIV latency, none of the other galectins (including those structurally similar to galectin-9, such as the other tandem-repeat type galectins, galectin-4 and galectin-8) exhibited any capacity to reactivate latent HIV infection at the same concentrations used for rGal-9, suggesting that HIV latency reversal is a unique feature of Gal-9 amongst members of the galectin family (Fig $1 \mathrm{~A}-1 \mathrm{C}$ ). Limiting duration of exposure of the J-Lat $5 \mathrm{~A} 8$ cell to $1000 \mathrm{nM}$ of rGal-9 for 6 hours achieved $\sim 67 \%$ of the activity observed after continuous 24 hours of treatment, while pulse treatment for only one hour achieved equivalent reactivation to $\alpha \mathrm{CD} 3 / \alpha \mathrm{CD} 28$ stimulation (S1 Fig). In addition, we examined the effects of $\mathrm{rGal}-9$ treatment on the expression of the HIV p24 and Vif proteins in J-Lat 5A8 cell lysates. Our data demonstrate that rGal-9 induces the expression of HIV viral proteins (S2 Fig).

\section{rGal-9 potently reverses HIV latency ex vivo}

We assessed the ability of rGal-9 to reactivate latent HIV in isolated CD4+ T cells derived from 13 HIV-infected individuals on suppressive ART. CD4+ T cells were treated with either DMSO $0.5 \%$ as negative control, rGal-9 at two different concentrations (500 $\mathrm{nM}$ and $1000 \mathrm{nM}$ ), PMA/ ionomycin $(2 \mathrm{nM}, 500 \mathrm{nM})$, or vorinostat $(1 \mu \mathrm{M})$ for 24 hours. $500 \mathrm{nM}$ and $1000 \mathrm{nM}$ of rGal-9 induced a mean 6.4-fold and 7.3-fold increase in intracellular HIV RNA levels after 24 hours, respectively, as compared to DMSO negative control $(\mathrm{p}=0.002)$. Induction was significantly higher than vorinostat $(\mathrm{p}=0.02,3.2 \mathrm{fold}$ ) (Fig 2A). Depleting CD4+ T cells expressing CD69, CD25, HLA-DR surface activation markers did not significantly affect rGal-9-mediated latent HIV reactivation ex vivo in CD4+ T cells, determined in a representative subset of three HIVinfected ART-suppressed individuals (Fig 2B and 2C). We additionally investigated the effects 
A

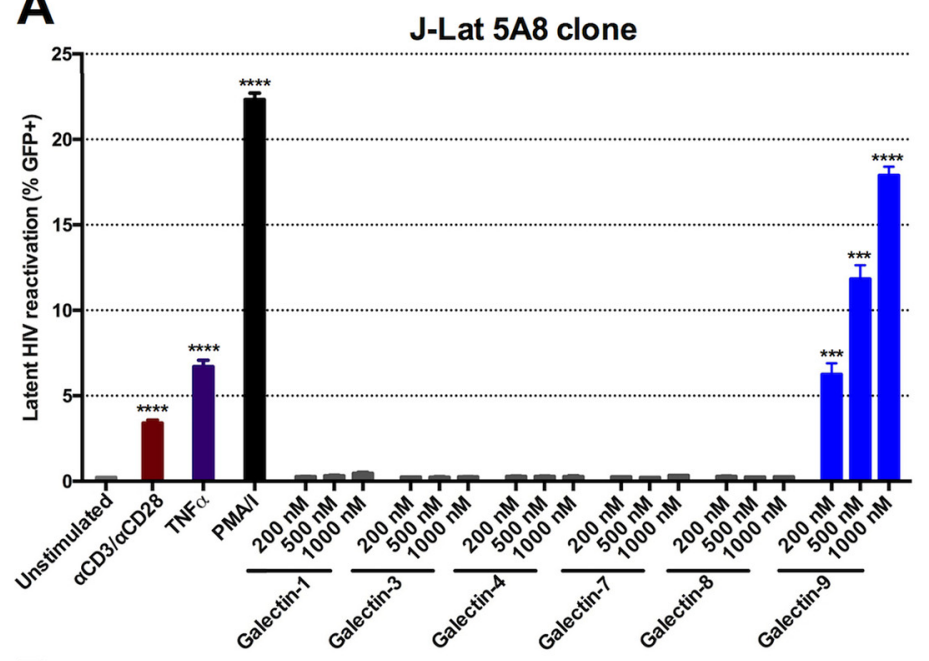

B
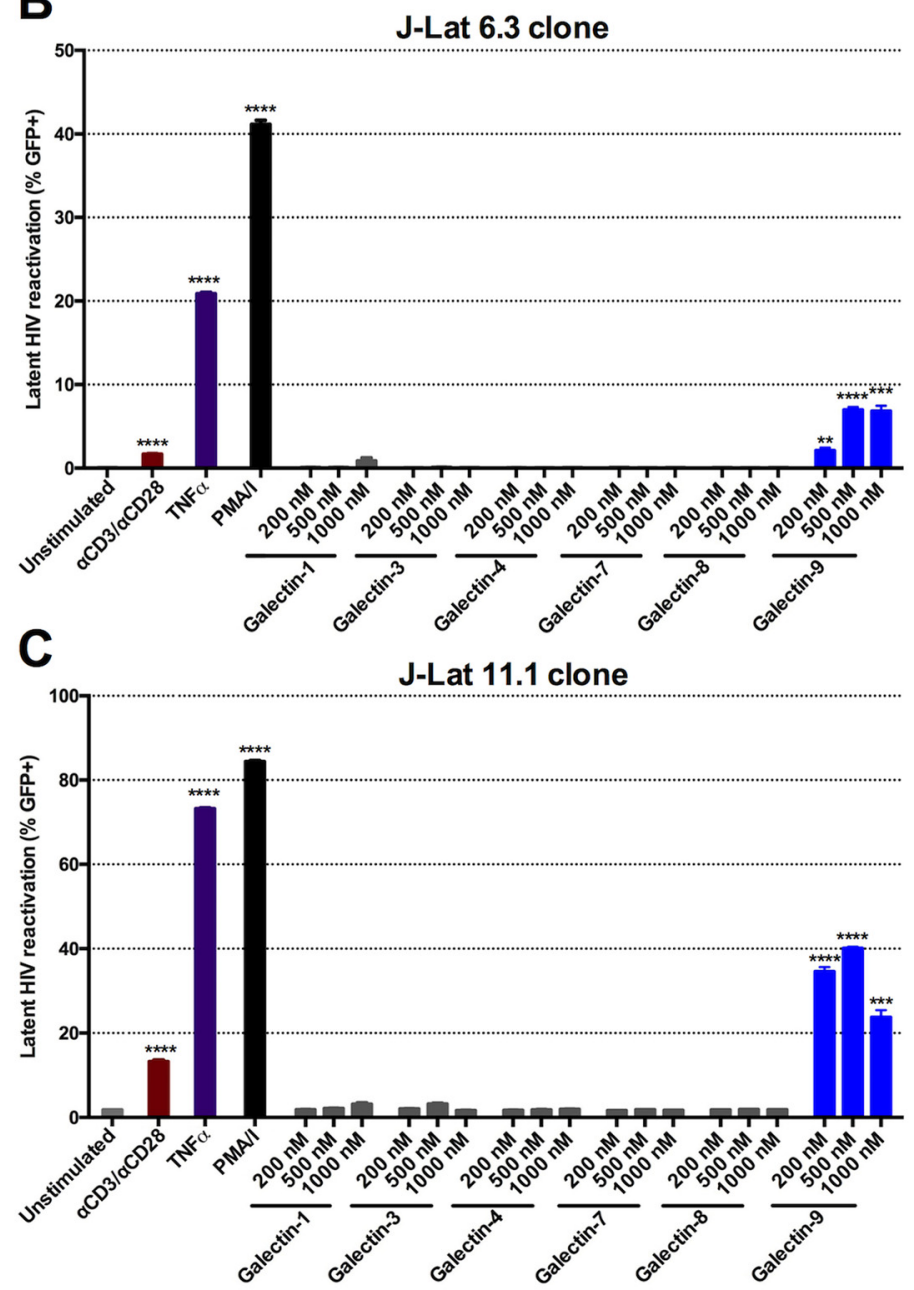

Fig 1. rGal-9 is a potent mediator of HIV transcription in vitro. in vitro HIV reactivation in the J-Lat latency model (A) 5 A8 clone, (B) 6.3 clone, and (C) 11.1 clone by varying doses of rGal-9 and other galectins (-1, -3, $-4,-7,-8$, and -9 ) after 24 hours of stimulation. $\alpha C D 3 / a C D 28$ antibodies conjugated to beads, PMA/ ionomycin $(16 \mathrm{nM} / 500 \mathrm{nM})$, and TNFa $(10 \mathrm{ng} / \mathrm{ml})$ were used as positive controls. J-Lat cells were analyzed by flow cytometry to assess HIV-encoded GFP expression. Mean \pm SEM is displayed, and statistical 
comparisons were performed using two-tailed unpaired t tests. ${ }^{*}=p<0.05 ;{ }^{*}=p<0.01,{ }^{* * *}=p<0.001$, and $* * * *=p<0.0001$

doi:10.1371/journal.ppat.1005677.g001
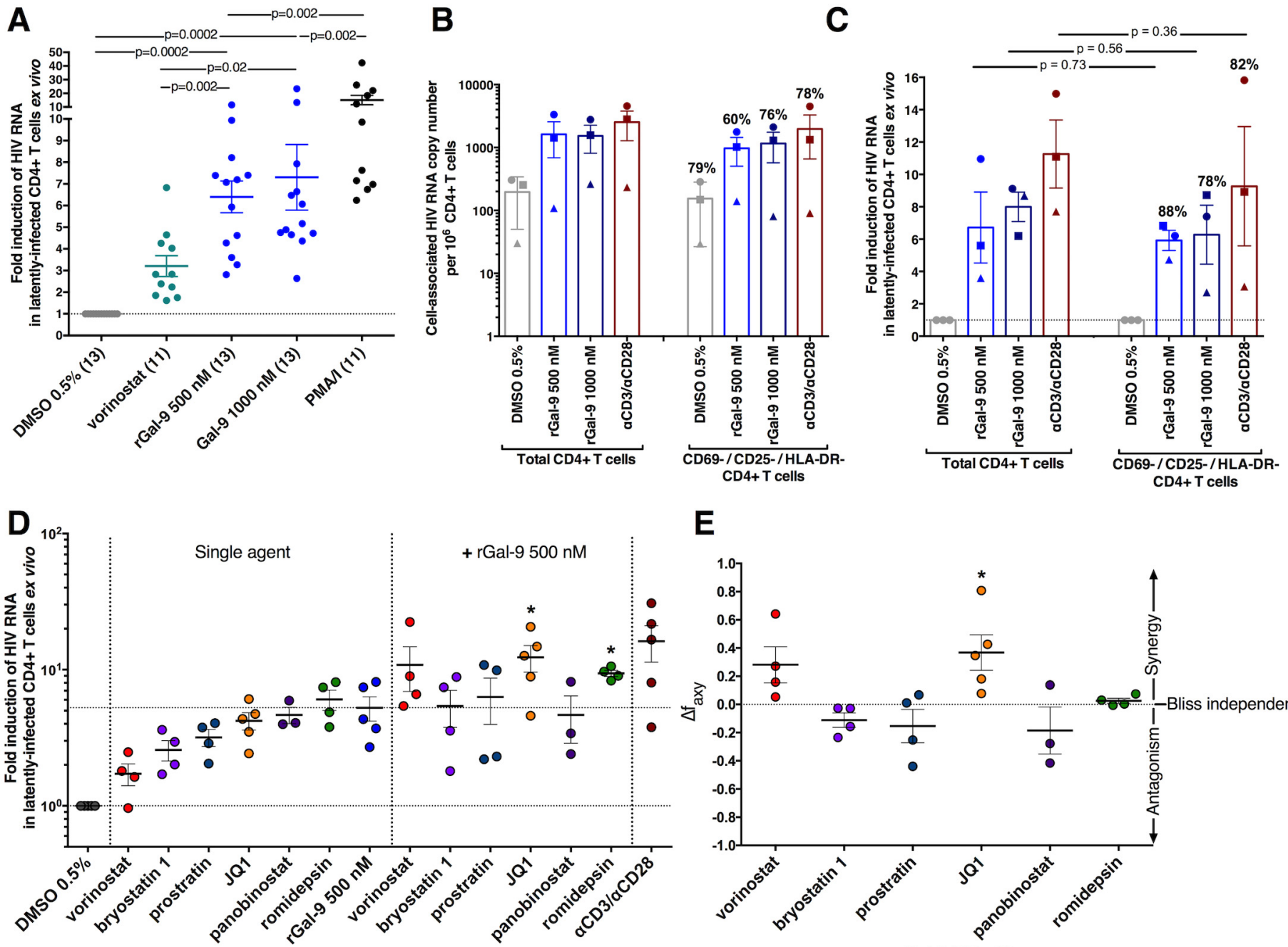

E

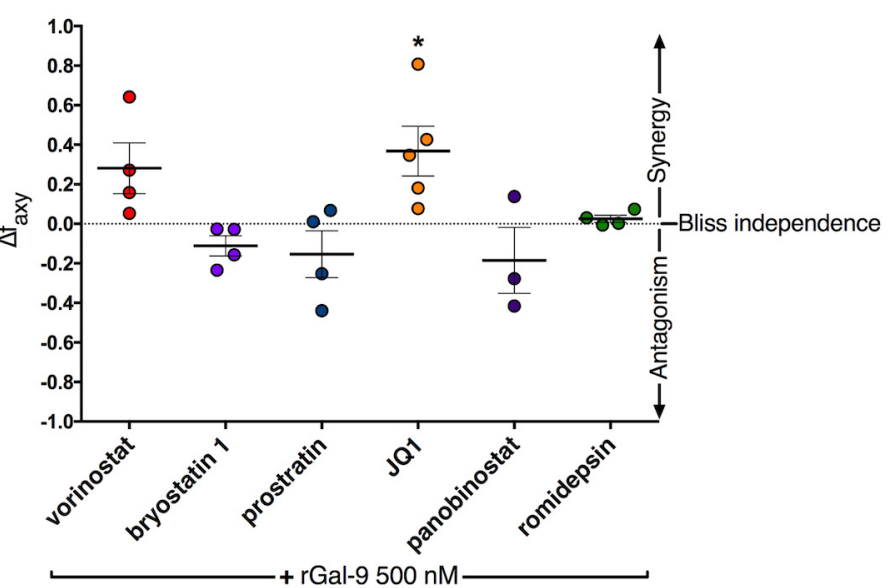

Fig 2. $\mathrm{rGal}-9$ is a potent mediator of HIV transcription ex vivo and synergizes with JQ1 in reactivating latent HIV. (A) Treatment of CD4+ T cells isolated from ART-suppressed HIV-infected individuals with DMSO $0.5 \%$ (negative control), PMA/ionomycin $(2 \mathrm{nM} / 500 \mathrm{nM})$, vorinostat $(1 \mu \mathrm{M})$, or varying concentrations of rGal-9 (500 nM and $1000 \mathrm{nM})$ for 24 hours. Fold increase in cell-associated HIV RNA was determined relative to the corresponding DMSO-treated control for each individual time point. Mean \pm SEM is displayed, and statistical comparisons between rGal-9 and other treatments were performed using two-tailed paired Wilcoxon signed-rank tests. (B-C) CD4+ T cells were isolated from PBMCs of three HIV-infected ART-suppressed individuals using negative selection. Resting CD4+ T cells were further enriched through depletion of cells expressing CD69, CD25, or HLA-DR surface markers from half of the isolated CD4+ T cells. The remaining half was processed through the exact enrichment procedure, except PBS was added instead

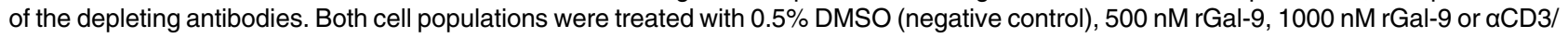
aCD28-conjugated beads. Induction of cell-associated HIV RNA was measured 24 hours post-treatment using RT-qPCR. Each individual is represented with a different symbol. Mean \pm SEM is displayed, and statistical comparisons were performed using two-tailed paired t tests. Percentages reported reflect average values measured in the CD69- / CD25- / HLA-DR- CD4+ T cells with respect to values observed in total CD4+ T cells. (D) CD4+ T cells from HIVinfected ART-suppressed individuals were treated with $500 \mathrm{nM}$ of rGal-9, $1 \mu \mathrm{M}$ vorinostat, $40 \mathrm{nM}$ romidepsin, $10 \mathrm{nM}$ bryostatin, $300 \mathrm{nM}$ prostratin, $1 \mu \mathrm{M}$ JQ1, or $30 \mathrm{nM}$ panobinostat alone or in combination with $500 \mathrm{nM}$ of rGal-9 for 24 hours, and fold induction of cell-associated HIV RNA was determined using quantitative real-time PCR. ${ }^{*}=p<0.05$ compared with $\mathrm{rGal}-9500 \mathrm{nM}$ treatment alone. (E) The Bliss independence model was utilized for calculation of synergy for drug combinations. $\Delta \mathrm{f}_{\mathrm{axy}}=0$ signifies a pure additive effect. $\Delta \mathrm{f}_{\mathrm{axy}}>0$ signifies synergy, while $\Delta \mathrm{f}_{\mathrm{axy}}<0$ signifies antagonism. Statistical significance was calculated using a two-tailed paired t-test comparing predicted and observed drug combination effects. ${ }^{*}=p<0.05$. 
of limited exposure ex vivo in the same subset of three HIV-infected ART-suppressed individuals. Our data demonstrate that six hours of exposure to $1000 \mathrm{~nm}$ of rGal-9 induces an average of $59.2 \%$ of the viral reactivation observed after 24 hours of continuous exposure ex vivo (S3 Fig).

We next explored synergy between rGal-9 and established latency reversal agents. In five HIV-infected ART-suppressed individuals, CD4+ T cells were treated with $500 \mathrm{nM}$ of rGal-9, $1 \mu \mathrm{M}$ vorinostat [7,10,11,14], $40 \mathrm{nM}$ romidepsin [14,37], $10 \mathrm{nM}$ bryostatin [14,38,39], $300 \mathrm{nM}$ prostratin [14,40-42], $1 \mu \mathrm{M} \mathrm{JQ1} \mathrm{[14,43-45],} \mathrm{or} 30 \mathrm{nM}$ panobinostat [14,46,47] alone or in combination with $500 \mathrm{nM}$ of rGal-9, in addition to $\alpha \mathrm{CD} 3+\alpha \mathrm{CD} 28$-conjugated beads (Dynal, at 1:1 bead:cell ratio) as positive control. Fold induction of cell-associated HIV RNA was determined using quantitative real-time PCR 24 hours after treatment. Based on the Bliss independence model for the quantitative analysis of synergy $[14,43,48]$, we determined that rGal-9 coadministration with the latency reversal agent "JQ1", a bromodomain inhibitor, exhibits synergistic activity $(\mathrm{p}<0.05)$ (Fig $2 \mathrm{D}$ and $2 \mathrm{E}$ ). In addition, we evaluated the effects of rGal-9 on the viability of freshly obtained primary CD4+ T cells from multiple HIV-infected ART-suppressed individuals. Our experiment demonstrated that $\mathrm{rGal}-9$ at high dosages (500 $\mathrm{nM}$ and $1000 \mathrm{nM}$ ) results in $15-20 \%$ reduction in cell viability, which is comparable to or better than existing LRAs such as romidepsin [37] (S4 Fig).

\section{rGal-9 reverses HIV latency in a glycan-dependent manner}

Published data have identified three $\mathrm{T}$ cell surface glycoproteins, $\mathrm{T}$ cell immunoglobulin and mucin protein-3 (Tim-3) [49], protein disulfide isomerase (PDI) [50], and CD44 [51], as receptors for Gal-9. Addition of anti-Tim-3 antibody, anti-PDI antibody, or anti-CD44 antibody prior to the addition of rGal-9 did not reduce rGal-9-mediated reactivation of latent HIV in $5 \mathrm{~A} 8$ cells. $\alpha$-lactose $(30 \mathrm{mM})$, an established neutralizer of Gal-9 activity [24], completely inhibited rGal-9-mediated reactivation of latent HIV (Fig 3A), while other saccharide derivatives (glucose and galactose at $30 \mathrm{mM}$ ) did not affect rGal-9 capacity for latency reversal (S5 Fig). Based on the known carbohydrate-binding nature of lectins, we investigated the requirement for complex $\mathrm{N}$ - and O-glycans in rGal-9-mediated HIV latency reversal. J-Lat 5A8 cells were treated for 24 hours with either tunicamycin or an enzymatic deglycosylation mix prior to rGal-9 stimulation. Tunicamycin is an antibiotic that chemically blocks $\mathrm{N}$-glycosylation of newly synthesized proteins at asparagine residues, and the deglycosylation mix enzymatically removes all $\mathrm{N}$-linked and O-linked carbohydrates from glycoproteins. rGal-9-mediated HIV latency reactivation (\%GFP+) was reduced from $14.1 \pm 0.6 \%$ to $0.3 \pm 0.03 \%$ (mean $\pm \mathrm{SD}$ ) when glycan synthesis was chemically inhibited by tunicamycin, or to $8.0 \pm 1.0 \%$ with enzymatic removal of cell surface glycans after 24 hours (Fig 3B and 3C). Conversely, the effects of PMA/ ionomycin on HIV latency reversal were enhanced by deglycosylation, while the activity of TNF $\alpha$ was unaffected (S6 Fig).

The enzymatic deglycosylation mix contains several enzymes (PNGase F, O-Glycosidase, $\alpha$ $(2 \rightarrow 3,6,8,9)$-Neuraminidase, $\beta(1 \rightarrow 4)$-Galactosidase, and $\beta$-N-Acetylglucosaminidase). We tested the ability of each enzyme individually or in combination to reduce the capacity of rGal9 to reverse HIV latency. A combination of PNGase F, $\beta(1 \rightarrow 4)$-Galactosidase, and $\beta$-N-Acetylglucosaminidase demonstrated the most potent reduction of rGal-9 mediated HIV reactivation as compared to non-deglycosylated cells $(14.7 \pm 1.2 \%$ to $7.9 \pm 0.1 \%)$ (Fig 3D). Thus, N-linked oligosaccharides and the less common, but widely distributed O-linked hexasaccharide structures $(\beta(1 \rightarrow 4)$-linked galactose and $\beta(1 \rightarrow 6)$-linked $\mathrm{N}$-acetylglucosamine) are essential ligands in rGal-9-mediated latency reversal. These data suggest that the ability of rGal-9 to reverse HIV latency depends on binding to a preferred set of glycan structures on the T cell surface membrane to transduce intracellular signals driving HIV transcription. 
A

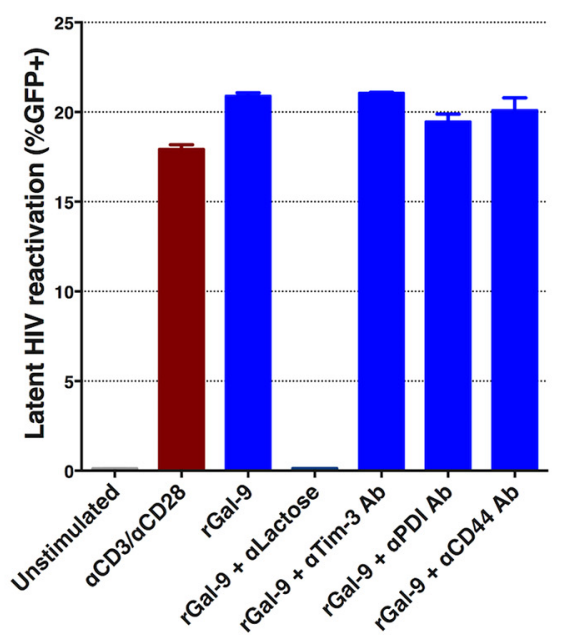

C

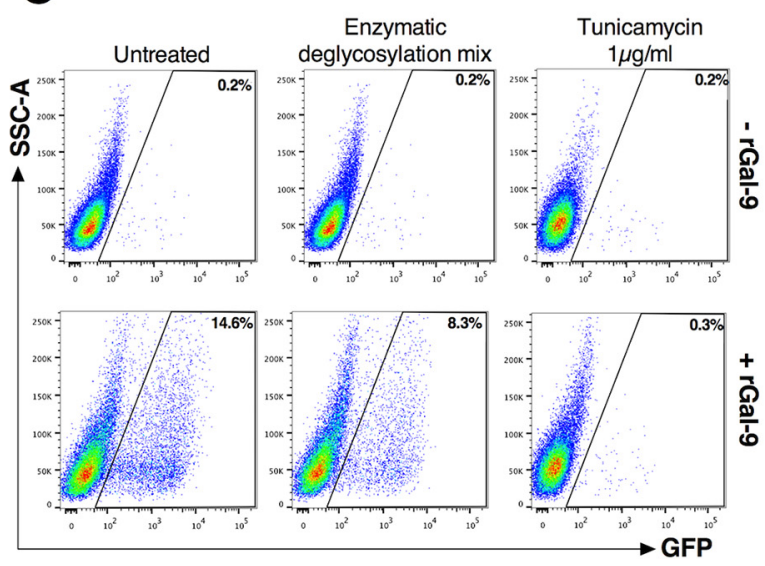

B

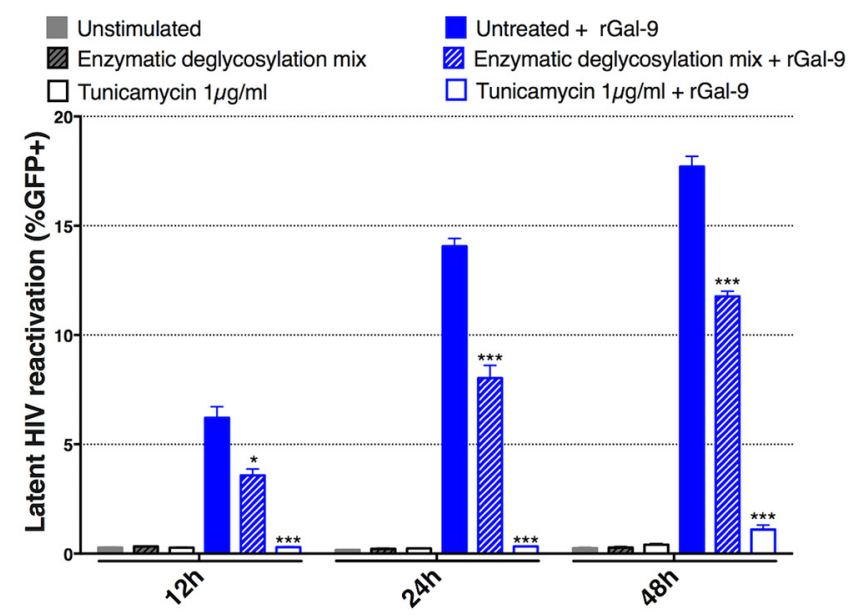

D

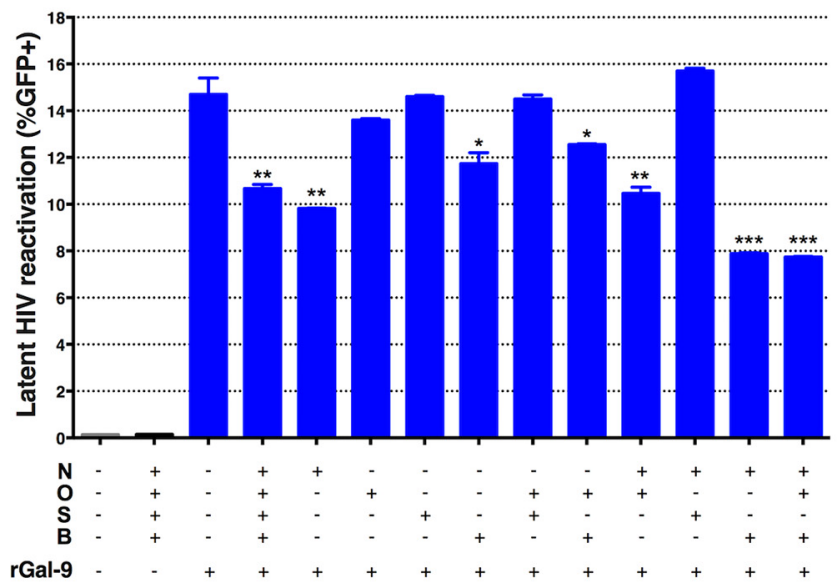

Fig 3. rGal-9 induces HIV transcription and reactivation in a glycan-dependent manner. (A) Effects of anti-Tim-3 antibody, anti-CD44 antibody, or anti-PDI antibody administration on rGal-9-mediated reactivation of HIV in J-Lat 5A8 cells. Antibodies were added 30 minutes prior to administration of $200 \mathrm{nM} \mathrm{rGal-9.} \alpha$-lactose $(30 \mathrm{mM})$ was used as a positive control. (B, C) Treatment of J-Lat 5 A8 cells with either $1 \mu \mathrm{g} / \mathrm{ml}$ tunicamycin, or with an enzymatic deglycosylation mix for 24 hours prior to rGal-9 stimulation. J-Lat cells were analyzed by flow cytometry to assess HIV-encoded GFP expression. Statistical comparisons were performed using two-tailed Mann-Whitney tests. (D)

Effects of deglycosylation enzyme combinations on rGal-9-mediated HIV latency reversal in J-Lat $5 A 8$ cells. $N=P N G a s e ~ F$ (Elizabethkingia miricola); $\mathrm{O}=\mathrm{O}$-Glycosidase (recombinant from Streptococcus pneumonia); $\mathrm{S}=\alpha-(2 \rightarrow 3,6,8,9)$-Neuraminidase (recombinant from Arthrobacter ureafaciens); $B=\beta(1 \rightarrow 4)$-Galactosidase (recombinant from Streptococcus pneumonia) $+\beta$ $\mathrm{N}$-Acetylglucosaminidase (recombinant from Streptococcus pneumonia). Mean \pm SEM is displayed, and statistical comparisons were performed using two-tailed unpaired t tests. ${ }^{*}=p<0.05 ;{ }^{*}=p<0.01,{ }^{* *}=p<0.001$, and ${ }^{* * *}=p<0.0001$.

doi:10.1371/journal.ppat.1005677.g003

rGal-9 modulates the gene expression of key transcription initiation, chromatin remodeling and promoter-proximal pausing factors that regulate HIV transcription

We implemented RNA-sequencing (RNA-seq) to identify a host gene expression signature associated with rGal-9-mediated HIV latency reversal. We examined sorted GFP-positive and GFP-negative cells containing reactivated (transcriptionally active) HIV proviruses and latent (transcriptionally inactive) proviruses, respectively, after stimulation with rGal-9, $\alpha \mathrm{CD} 3$ / 
$\alpha \mathrm{CD} 28$, or a combination of both agents. Cells with transcriptionally active proviruses (GFP+) as a result of rGal-9 stimulation exhibited a distinct gene expression pattern, compared to cells with transcriptionally active proviruses as a result of $\alpha \mathrm{CD} 3 / \alpha \mathrm{CD} 28$ stimulation (Fig $4 \mathrm{~A}, \mathrm{~S} 7$ Fig). We determined that $\mathrm{rGal}-9$ significantly modulates the expression of genes involved in several established signaling pathways that are known to play important roles in maintaining HIV latency (FDR $<0.05$ ) (Fig 4B). rGal-9 induces the gene expression of several HIV proviral transcription initiation factors including the nuclear factor kappa-light-chain-enhancer of activated $\mathrm{B}$ cells (NFkB), AP-1 nuclear complex, and the calcium-dependent activator of HIV transcription initiation (NFAT). In addition, $\mathrm{rGal}-9$ inhibits the gene expression of several chromatin modification and remodeling factors, including histone deacetylase 1,2, and 3, EZH2, SUV39H1, DNMT1, BAF complex, and BCL11B. Lastly, rGal-9 inhibits the gene expression of the promoter-proximal pausing factors belonging to the NELF family.

\section{rGal-9 partially activates primary CD4+ T cells and induces naïve CD4+ T cell proliferation}

In addition to evaluating the effects of rGal-9 on latent HIV reactivation, we sought to determine the effects of rGal-9 on primary CD4+ T cell phenotype, focusing on CD4+ T cell activation, proliferation and apoptosis. $500 \mathrm{nM}$ and $1000 \mathrm{nM}$ of rGal-9 induced surface expression of the CD69 activation marker (73.9\%, 81.8\%, respectively), and marginally induced CD25 (4.6\%, $6.4 \%$, respectively) (Fig 5A and 5B) on the surface of isolated CD4+ T cells from six HIVinfected ART-suppressed individuals. $200 \mathrm{nM}$ and $500 \mathrm{nM}$ of rGal-9 resulted in proliferation of primary CD4+ T cells isolated from three HIV-infected ART-suppressed individuals after five days of culture (10.3\%, 12.6\%, respectively) (Fig 5C), as measured by CFSE staining. rGal-9 selectively induced proliferation of naïve CD4+ T cells (CD4+ CD45RA+) $(12.6 \%, 13.7 \%$, respectively) rather than memory $\mathrm{CD} 4+\mathrm{T}$ cells (CD4+ CD45RA-) (3.1\%, 4.9\%, respectively) (Fig 5E and 5F). In accordance with our ex vivo phenotypic data, we observed that rGal-9 modulates the expression of a subset of genes associated with regulation of $\mathrm{T}$ cell activation and proliferation in vitro (S8A-S8C Fig), and up-regulates multiple genes responsible for T cell apoptosis (S8E Fig).

\section{rGal-9 induces the expression of several anti-HIV host restriction factors including APOBEC3G}

Achieving reservoir clearance or sustained virologic control of HIV following LRA administration will likely require involvement of innate and intrinsic immunity [8]. We therefore sought to determine the effects of rGal-9 on the expression of genes involved in innate immunity, cellintrinsic immunity, and cytokine production (S9 Fig). We implemented a qPCR array to measure the expression of 42 established anti-HIV host restriction factors in vitro (in J-Lat 5A8 cells) and ex vivo (in primary CD4+ T cells isolated from ten ART-suppressed HIV-infected individuals). rGal-9 significantly modulates the expression of several established anti-HIV cellintrinsic immune defenses. Of particular relevance, we determined that the APOBEC 3 cytidine deaminases, including APOBEC3G, were potently induced by rGal-9 in vitro (up to 55 fold) (S10 Fig). This induction was confirmed by targeted digital RT-dPCR at the mRNA level (Fig 6A-6C) and by western blotting at the protein level (Fig 6D and 6E). In addition, we determined that APOBEC3G expression was induced by $\mathrm{rGal}-9$ ex vivo $(\mathrm{p}=0.016)$ in $\mathrm{CD} 4+\mathrm{T}$ cells from HIV-infected ART-suppressed individuals at the mRNA level (up to 14 fold) using qPCR (Fig 7A and 7B, S11 Fig) and at the protein level using western blotting (Fig 7C and 7D). In contrast to the induction observed in response to rGal-9 treatment, APOBEC3G expression was significantly reduced by vorinostat treatment ex vivo $(\mathrm{p}=0.016)$ (Fig $7 \mathrm{~A}$ and $7 \mathrm{~B})$. 
A

B

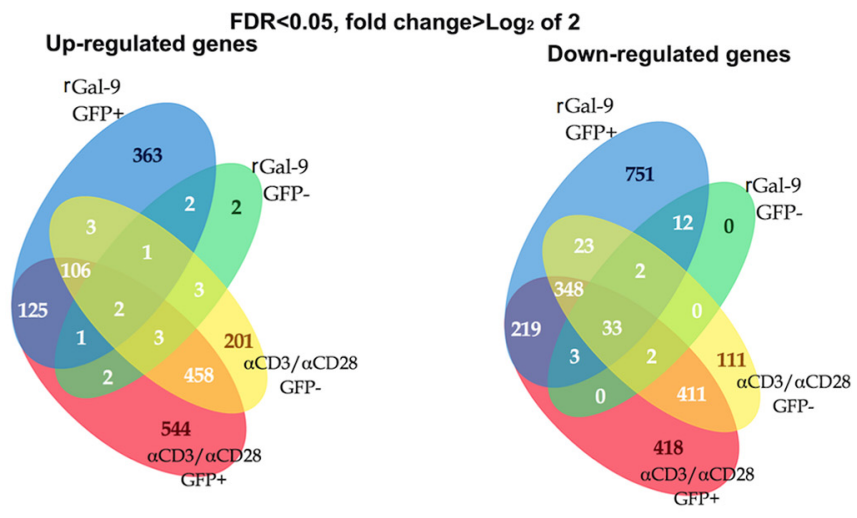

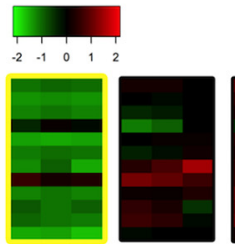

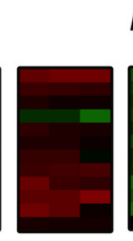

$N F-K B$
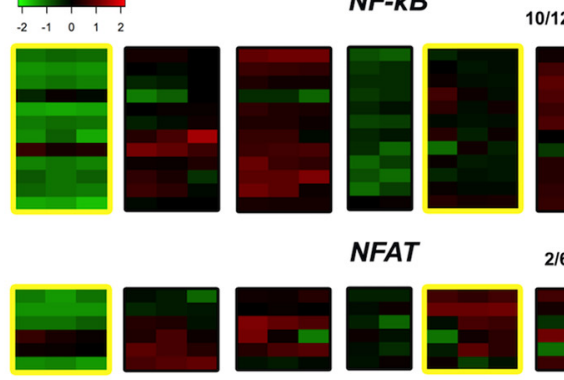

NFAT
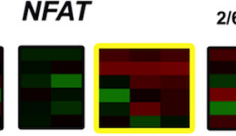

AP-1
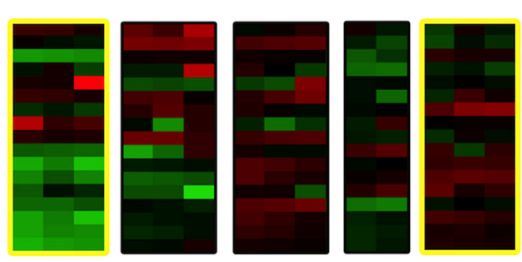

HDACs
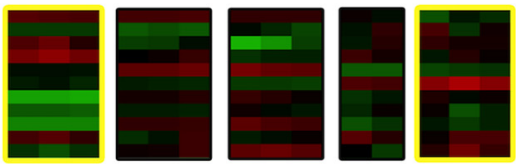

$4 / 11$
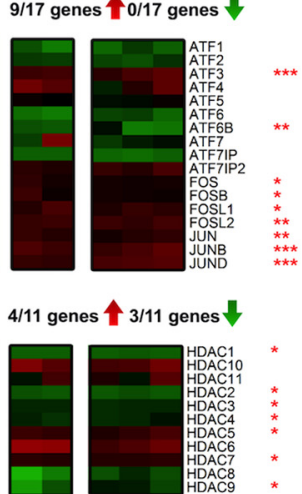

$B A F$
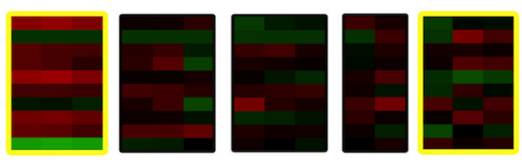

1/10 genes 4/10 genes
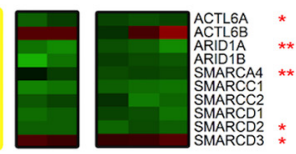

NELF

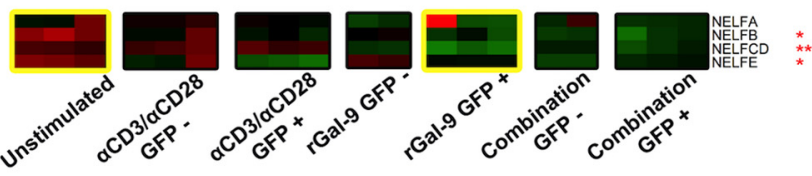

Fig 4. rGal-9 modulates the expression of genes involved in several signaling pathways associated with HIV latency. (A) Venn diagram showing the number of genes modulated by $>2$ fold with FDR $<0.05$ in sorted GFP-positive and GFP-negative cells containing reactivated (transcriptionally active) HIV proviruses, and latent (transcriptionally inactive) proviruses, respectively, after either rGal-9 treatment or $\alpha C D 3 / \alpha C D 28$ stimulation. (B) Heat maps describing effects of $r G a l-9$ treatment on host gene expression, organized by signaling pathways. All statistical comparisons were performed using $t$ tests, and $p$ values were adjusted for multiple comparisons using false 
discovery rate. Asterisks indicate $>2$-fold, statistically significant differences in gene expression between $\mathrm{r}$-Gal9-treated, GFP+cells and unstimulated control, as follows: ${ }^{*}=$ FDR $<0.05 ;^{*}=$ FDR $<0.01$, and ${ }^{* * *}=$ FDR $<0.001$.

doi:10.1371/journal.ppat.1005677.g004

\section{rGal-9 significantly reduces infectivity of progeny virus}

APOBEC $3 \mathrm{G}$ is an innate antiviral factor that is incorporated into virions and drives extensive G-to-A mutation, or "hypermutation", of the HIV genome, typically rendering it non-viable within a single replicative cycle $[52,53]$. We therefore hypothesized that latent virus that is reactivated by rGal-9 (in the setting of elevated APOBEC3G expression) will likely be hypermutated and rendered replication incompetent upon infection of a new cell. To test the hypothesis that virus produced in the presence of rGal-9 is associated with reduced infectivity, we spinoculated MOLT4-CCR5 cells with replication-competent HIV for two hours. After six hours of incubation, cells were washed and were treated with PBS, $200 \mathrm{nM}$ of rGal-9, or $5000 \mathrm{U} / \mathrm{ml}$ of interferon- $\alpha$ for 24 hours, followed by incubation for three days (Fig $8 \mathrm{~A}$ ). Culture supernatants were concentrated and aliquots of the concentrated culture supernatants were used to measure HIV production by quantifying viral p24 antigen concentration (Fig 8B), and equal amounts of the remaining culture supernatants were used to infect Jurkat cells (by spinoculation) for 12 days. Alu-qPCR was used to quantify the levels of integrated HIV in the Jurkat target cells every 3 days (Fig $8 \mathrm{~A}$ ). rGal-9 treatment of producer cells resulted in a 7.1-fold reduction in the level of integrated HIV DNA in target cells (normalized to producer cell p24 supernatant levels) after 3 days of culture. This reduction remained stable up to 12 days (6.9 fold at day 6, 6.3 fold at day 9, and 12.1 fold at day 12 ) (Fig $8 \mathrm{C}$ and $8 \mathrm{D}$ ). These data suggest that a significant fraction of the viral particles produced by MOLT4-CCR5 cells treated with rGal-9 were defective in a manner that prevented successful integration into the host genome.

\section{Soluble galectin-9 (sGal-9) levels in plasma of HIV-infected ART- suppressed individuals are associated with HIV transcription in vivo}

Based on our data that rGal-9 potently induces HIV transcription in vitro and ex vivo, we hypothesized that endogenous sGal-9 regulates HIV transcription in vivo, in HIV-infected, ART-suppressed individuals. We examined relationships between plasma levels of sGal-9 in 72 HIV-infected individuals on suppressive ART and levels of CD4+ T cell-associated HIV RNA (marker of HIV transcription), and the quantity and binding avidity of HIV-specific antibodies, which have been associated with HIV production in multiple studies [4,54,55]. Levels of sGal-9 were positively correlated with levels of CD4+ T cell-associated HIV RNA ( $p=0.02$, Spearman $r=0.27$ ), as well as with the quantity and binding avidity of anti-HIV antibodies $(p=0.0005$, Spearman $r=0.39$ ) (Fig 9A and 9B). These data suggest that endogenous Gal-9 likely plays an important role in regulating HIV transcription and viral production during suppressive therapy.

\section{Discussion}

In this study, we have revealed a novel biological function of lectins and cell surface carbohydrates in the biology of viral latency. Our findings strongly suggest that manipulation of Gal-9 (via modulation of endogenous production or exogenous administration of recombinant protein) should be explored as a foundation for novel HIV curative strategies. A large body of literature demonstrates that recombinant Gal-9 is well-tolerated and exhibits therapeutic potential for a range of disorders in animal models [27-31], reinforcing its potential as a therapeutic tool for HIV infection. Furthermore, the observed synergy with the bromodomain inhibitor JQ1 suggests that Gal-9 may serve as a useful component of multi-drug HIV eradication cocktails. 
A

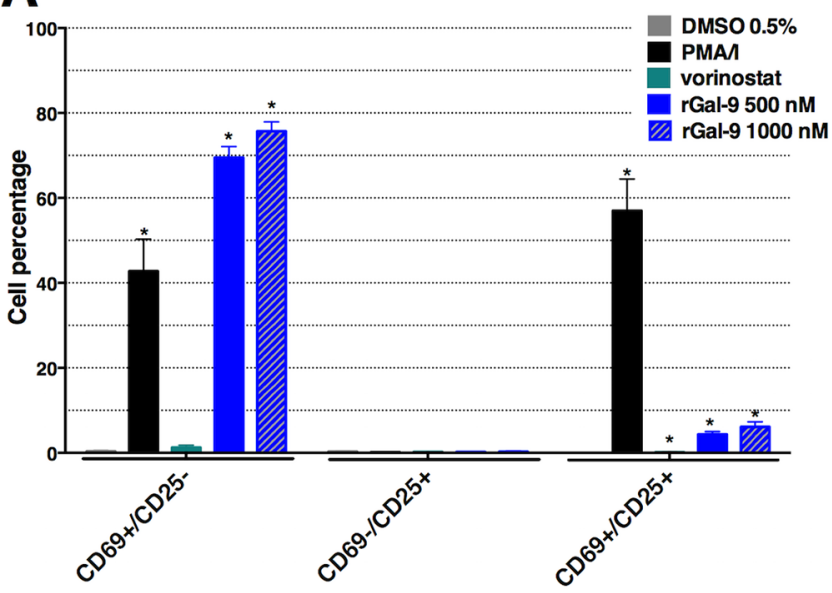

C

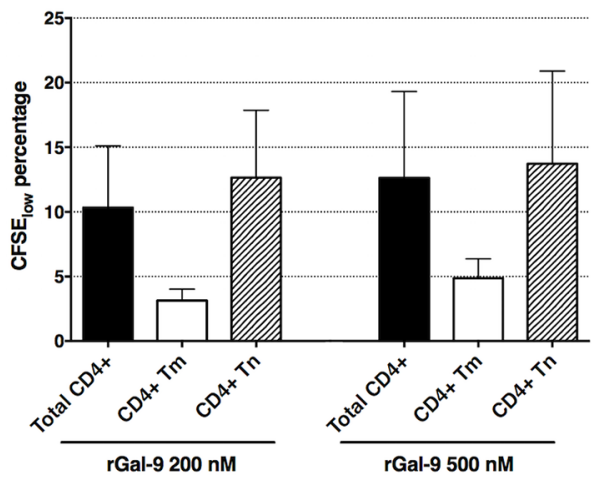

E

Memory CD4+ $T$ cells $(\mathrm{Tm})$

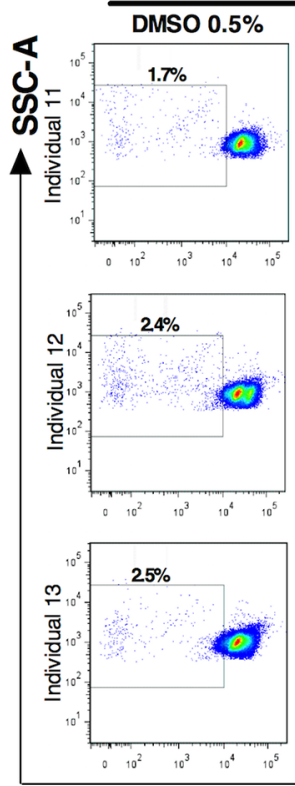

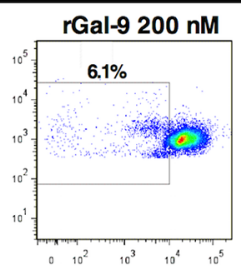
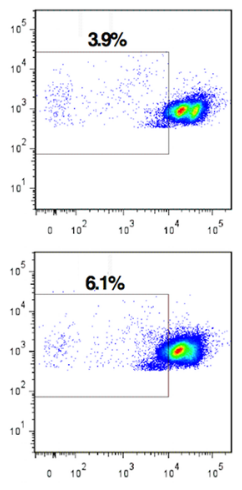

B
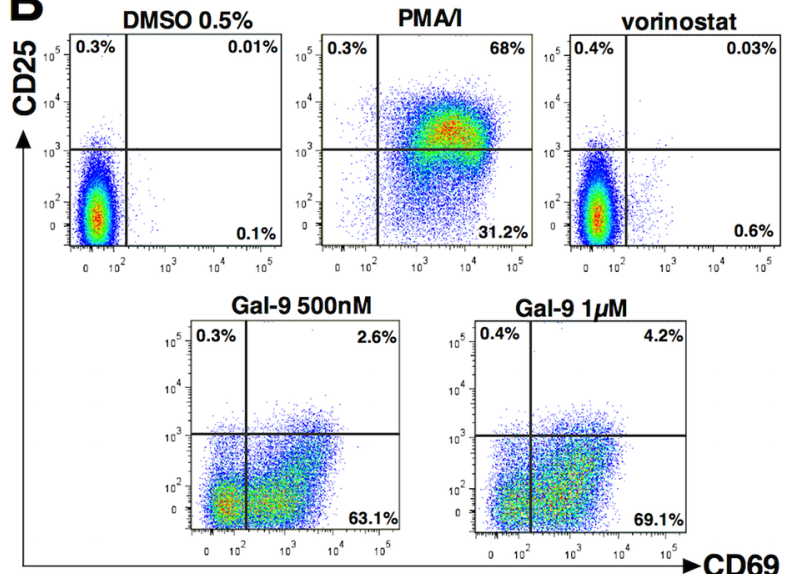

$\rightarrow \mathrm{CD} 69$

D

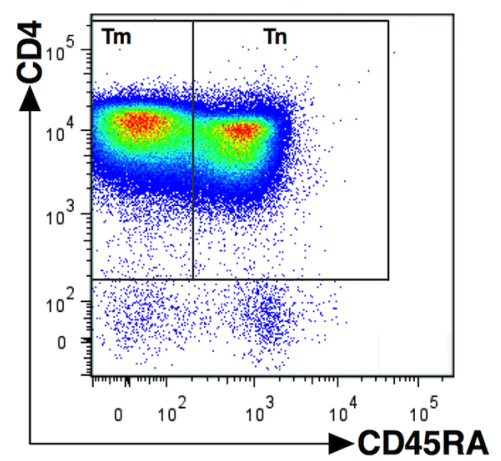

F

Nalive CD4+ $T$ cells $(T n)$
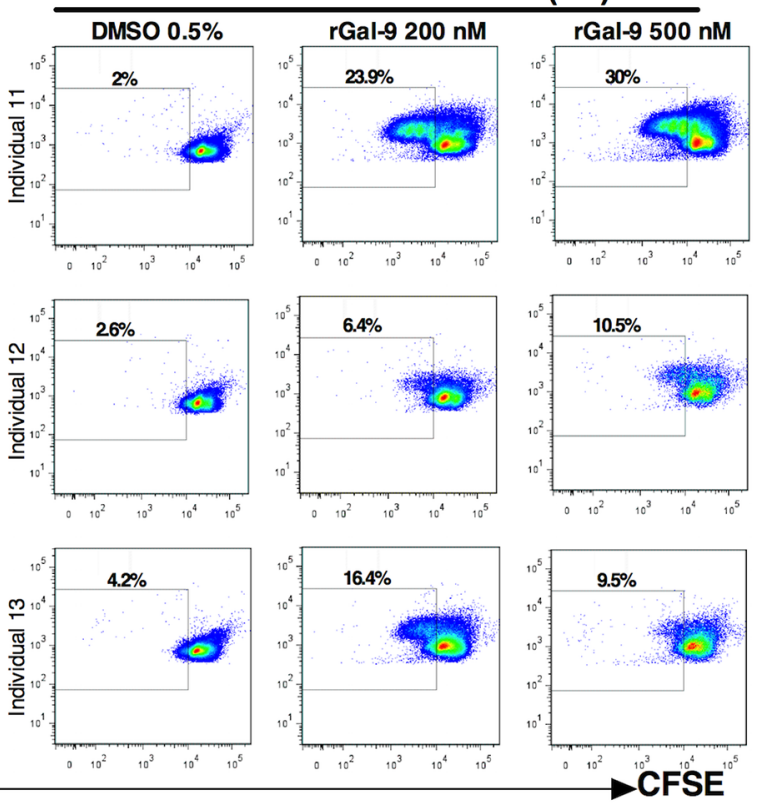

Fig 5. rGal-9 partially activates primary CD4+ T cells and induces proliferation primarily in naïve CD4+ T cells. (A, B) Effects of rGal-9 stimulation on the cell surface expression of CD69 and CD25 activation markers on CD4+ T cells isolated from six ARTsuppressed individuals. Mean \pm SEM is displayed. Asterisks represent statistically significant differences as compared to DMSO control $(p<0.05$, two-tailed Wilcoxon signed-rank test). (C) Effects of rGal-9 stimulation on the proliferation of CD4+ T cells isolated from three ART-suppressed individuals. Primary CD4+ T cells were stained with CFSE and cultured for 5 days, stained with CD4 and CD45RA 
monoclonal antibodies, and proliferation was quantified as the percentage of CFSE $\mathrm{Iow}_{\mathrm{low}}$ cells on CD4+ CD45RA+ (Naïve, Tn) or CD4 + CD45RA- (Memory, Tm) T cells. Mean \pm SEM is displayed. (D) Example of the flow cytometry gating strategy. (E, F) Effects of rGal-9 on proliferation of $(E)$, memory CD4+ T cells, and (F), naïve CD4+ T cells.

doi:10.1371/journal.ppat.1005677.g005

Galectins recognize galactose-containing saccharide sequences displayed on cell surface glycoconjugates [21]. Activated CD4+ T cells and HIV latently-infected cells exhibit an altered cell surface glycosylation pattern with respect to resting, uninfected cells [56]. This aberrant pattern includes loss of sialic acid and an increase of core $2 \mathrm{O}-$ Glycans [56]. In addition, the association between hyposialylation and HIV infection has been established in vivo [57,58]. It has been further demonstrated that the loss of sialic acid promotes oligosaccharide binding to Gal-9 and promotes Gal-9 activity [59]. Collectively, these data suggest that the effects of Gal-9 may be more pronounced in HIV latently-infected cells than in uninfected cells. This specificity may allow for potent viral reactivation and reservoir clearance in vivo with minimal undesirable perturbation of uninfected cells. Additional studies are needed to decipher the nature of glycan-mediated recognition responsible for Gal-9-mediated signal transduction.

The APOBEC 3 cytidine deaminases, including APOBEC3G and APOBEC3F, were strongly induced by rGal- 9 in CD4+ T cells. Based on the established mechanism of action associated with the APOBEC 3 factors, the pronounced APOBEC 3 induction by rGal-9 would be expected to override the antagonistic activity of the HIV Vif protein $[60,61]$ and result in hypermutation of progeny virus upon infection of a new cell, rendering the virus replication incompetent. This phenomenon is supported by our observation that rGal-9 treatment reduces viral infectivity by over seven-fold. This detail is highly relevant to the shock-and-kill HIV cure framework, as it is becoming increasingly clear that ART does not completely block viral replication, especially in tissues with suboptimal drug penetration [62]. APOBEC3 induction in the producer cell will reduce the probability that the HIV reservoir is replenished when latency is reversed therapeutically. This pattern is in stark contrast to our observation that vorinostat significantly suppresses expression of APOBEC3G in CD4+ T cells. This unintended effect of vorinostat may contribute to the published observation that vorinostat enhances cellular susceptibility to HIV infection [63].

The involvement of host immunity will be critical in achieving clearance of the latent HIV reservoir. On this front, $\mathrm{rGal}-9$ was previously found to have immunopotentiating effects [64]. rGal-9 enhanced cytotoxic $T$ cell activity against tumor cells, enhanced IFN- $\gamma$ and IL-4 production, and promoted dendritic cell maturation via Gal-9-Tim-3 interactions. Our CFSE staining data demonstrate that $\mathrm{rGal}-9$ induces $\mathrm{T}$ cell proliferation, compatible with immunopotentiating activity. Although induction of proliferation by an LRA may inadvertently expand the latent reservoir, it is important to note that proliferation driven by rGal-9 was observed primarily in the naïve $\mathrm{CD} 4+\mathrm{T}$ cell compartment rather than in memory CD4+ T cells. Multiple recent studies have shown that naïve CD4+ T cells rarely harbor latent HIV[65]. Therefore, the induction of CD4+ T cell proliferation by rGal-9 is unlikely to significantly contribute to the expansion of the latent pool. Other studies have demonstrated immunosuppressive effects of rGal-9 in the setting of autoimmune arthritis and organ transplantation rejection models [66,67]. Taken together, it is likely that rGal-9 exhibits immunopotentiating activity in the setting of immunosuppression, and immunosuppressive activity in the setting of hyperimmunity. The effect of rGal-9 on cell-mediated immunity against HIV warrants proper investigation in animal models and clinical studies. Although in vitro methods are available to investigate the effects of rGal-9 on innate and adaptive immune responses, recent reports demonstrate that the effects of LRAs on anti-HIV immunity in vitro do not accurately reflect the immunologic consequences of administration in vivo $[13,68]$. 


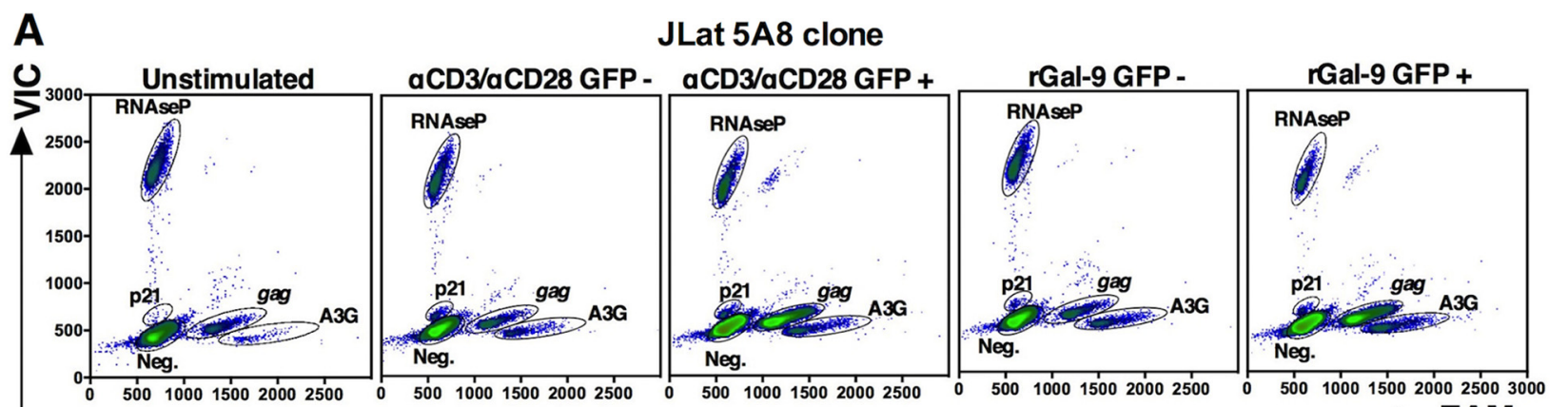

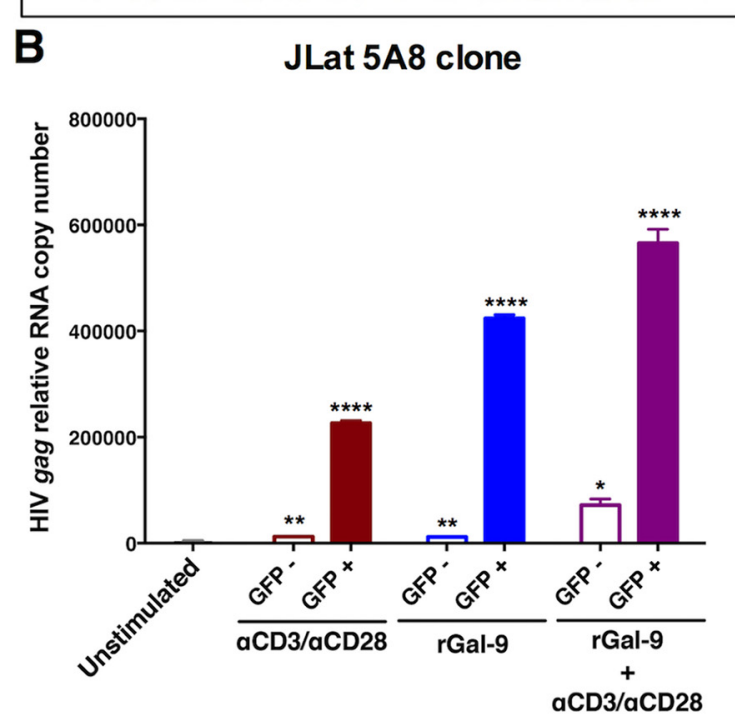

D

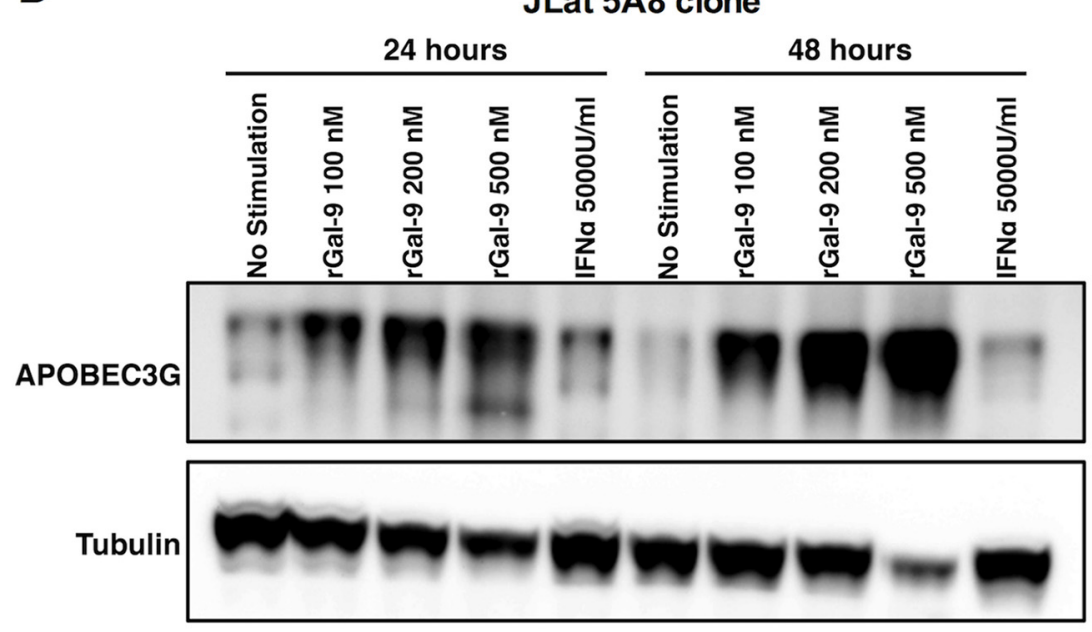

FAM

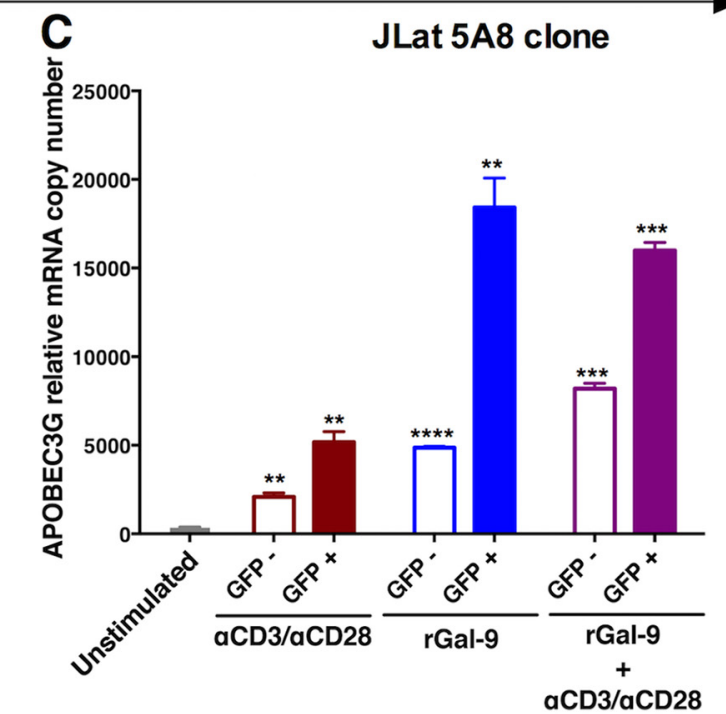

\section{E JLat 5A8 clone}

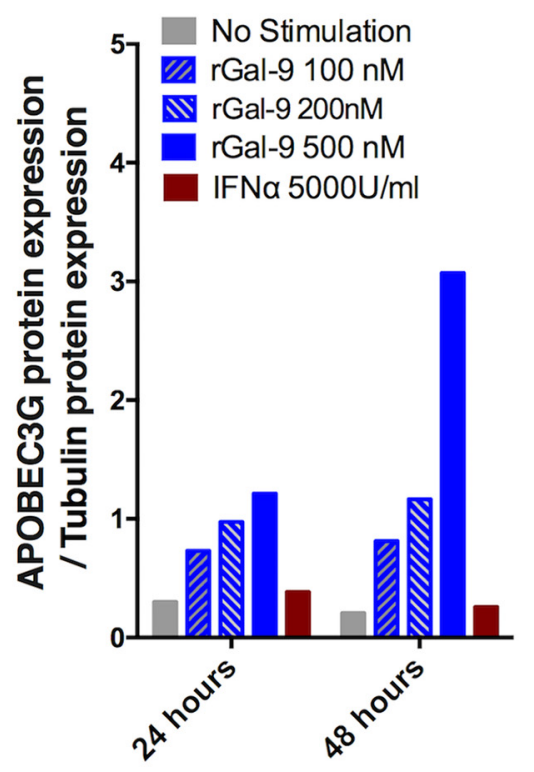

Fig 6. rGal-9 induces the expression of the APOBEC3G anti-HIV host restriction factor in vitro. (A-C) Digital droplet PCR gene expression profiling quantifying HIV gag, host APOBEC3G, p21, and RNAseP (housekeeping control) mRNA in J-Lat 5A8 cells sorted into GFP-positive and GFP-negative populations after either rGal-9 treatment, aCD3/aCD28 stimulation, or a combination of both. Mean \pm SEM is displayed, and statistical comparisons against the unstimulated control were performed using two-tailed unpaired $t$ tests. ${ }^{*}=p<0.05 ;{ }^{* *}=p<0.01,{ }^{* *}=p<0.001$, and ${ }^{* * *}=p<0.0001$. (D-E) APOBEC3G protein expression in J-Lat 5 A8 cells treated with 
varying concentrations of rGal-9 $(100 \mathrm{nM}, 200 \mathrm{n} \mathrm{M}$, and $500 \mathrm{nM})$ or interferon- $\alpha$ (5000 units $/ \mathrm{ml})$, as determined by western blot. Immunoblotting bands were quantified with ImageJ software. The quantified APOBEC3G protein expression levels were normalized to corresponding Tubulin protein levels to account for variation in loading.

doi:10.1371/journal.ppat.1005677.g006

In summary, our study provides the first evidence that Gal-9 should be considered as a foundation for novel lectin-based strategies to eliminate the latent HIV reservoir. Detailed exploration of biochemical pathways associated with Gal-9 and implementation of animal models will be critical in translating our findings into novel therapeutic or curative approaches for HIV infection.

\section{Materials and Methods}

\section{Ethics statement}

This study utilized retrospectively and prospectively collected specimens from HIV-infected individuals enrolled in the UCSF SCOPE and Hawaii HIV cohorts. Research protocols were approved by the relevant University of California, San Francisco and University of Hawaii Committees on Human Research. All participants were enrolled after obtaining written informed consent, and all subject data and specimens were coded to protect confidentiality.

\section{Subjects and specimen processing}

One milliliter ( $\mathrm{ml}$ ) of plasma was collected retrospectively from 72 ART-suppressed HIVinfected individuals enrolled in the University of California, San Francisco (UCSF) SCOPE and Options cohorts who have been on ART for 1-2 years at time of sampling. We previously measured levels of cell-associated HIV RNA in CD4+ T cells from the same 72 individuals [17,69]. We prospectively recruited 10 participants from the SCOPE cohort. Participants met strict selection criteria and had well-documented persistent viral suppression for over 3 years. This study was approved by the UCSF Committee on Human Research. Fresh blood (100 ml) was collected and peripheral blood mononuclear cells (PBMCs) were isolated from whole blood using SepMate (STEMCELL Technologies). CD4+ T cells were isolated from PBMCs using negative selection (EasySep, STEMCELL Technologies). In addition, one billion cryopreserved PBMCs were collected from five ART-suppressed HIV-infected individuals enrolled in the Hawaii HIV-1 cohort (HHC). Subject characteristics for all 15 participants involved in our prospective sample collection are documented in detail in S1 Table. The characteristics of the 72 ART-suppressed HIV-infected individuals samples used to measure soluble levels of Gal-9 are described in detail in our previous publications $[17,69]$. CD4+ T cells were enriched from the cryopreserved PBMCs by negative selection using the EasySep Human CD4+ T Cell Enrichment Kit (Stemcell Technologies), according to the manufacturer's instructions.

\section{Recombinant and natural galectins}

Stable forms of recombinant galectins 1 and 9 were obtained through our collaborators at GalPharma Co., Ltd. (Kagawa, Japan). Galectin-1 and galectin-9 are very unstable in nature, hence stable forms with retaining biological activities were used. For the other galectins $(-3,-4,-7$, and, -8$)$, the natural, stable form was used [70,71].

\section{Measurement of HIV latency reversal in vitro}

We performed experiments in the established "J-Lat" model of HIV latency (kindly provided by Drs. Warner Greene and Eric Verdin, Gladstone Institute of Virology and Immunology). J-Lat cells harbor latent, transcriptionally competent HIV provirus that encodes green 
A

\section{Primary CD4+ T cells}

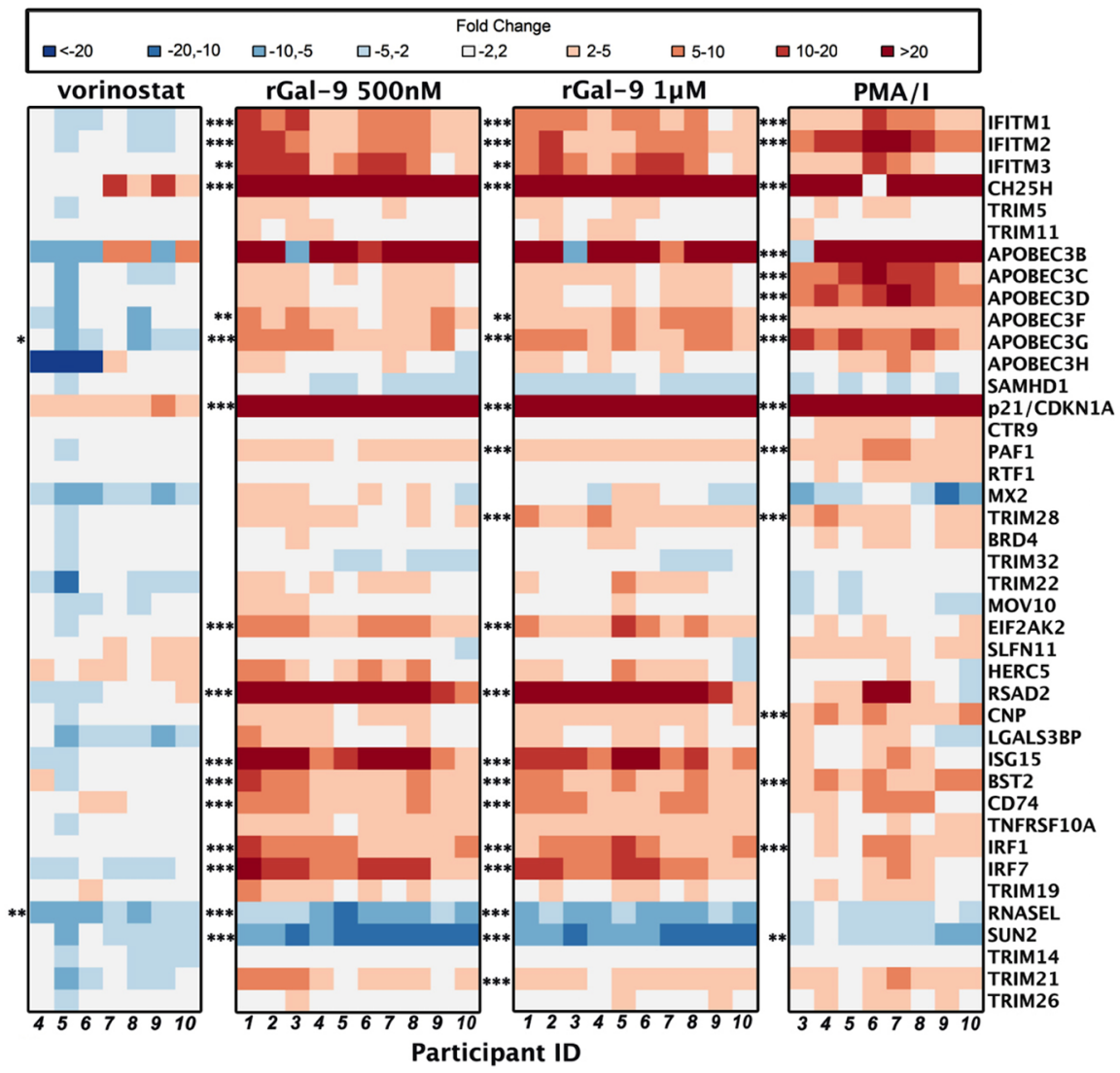

B

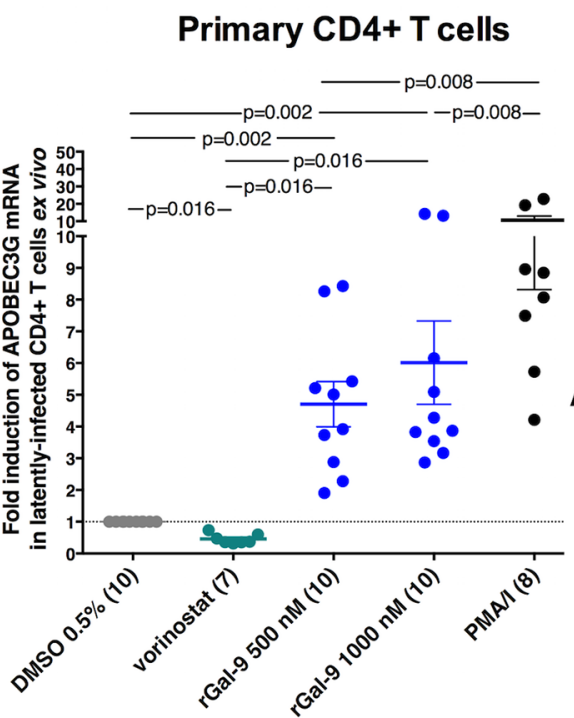

C

Primary CD4+ T cells

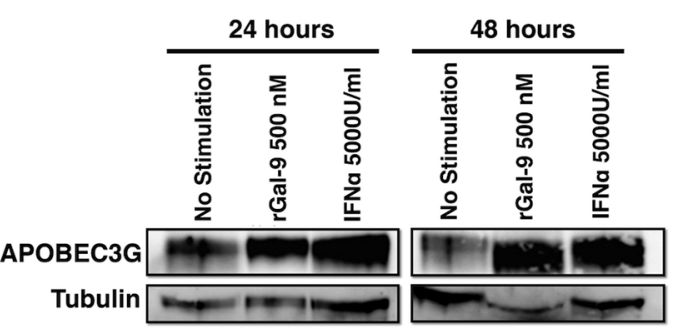

Tubulin
D

Primary CD4+ T cells

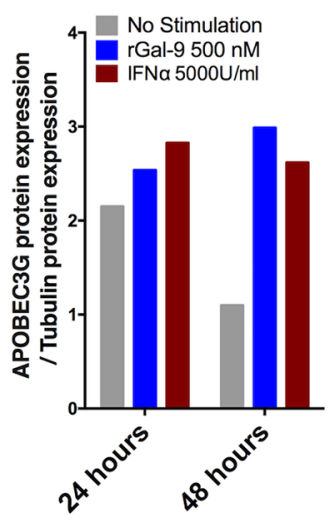

Fig 7. rGal-9 induces the expression of several anti-HIV host restriction factors including APOBEC3G ex vivo. (A) Heat map representing expression levels of host restriction factors in CD4+ T cells isolated from ART-suppressed individuals, after treatment with either $0.5 \%$ DMSO as negative control, $500 \mathrm{nM}$ rGal-9, $1000 \mathrm{nM}$ rGal-9, $1 \mu$ M vorinostat, or a combination of PMA (2 nM) and lonomycin $(0.5 \mu \mathrm{M})$. Heat colors indicate fold modulation compared to the DMSO control. Red indicates induction of expression, and blue indicates reduction of expression. Statistical comparisons were performed using $t$ tests, and $p$ values were adjusted for multiple 
comparisons using false discovery rate. Asterisks indicate $>3$-fold, statistically significant modulation of gene expression as compared to DMSO control, as follows: ${ }^{*}=$ FDR $<0.05 ; * *=F D R<0.01$, and $* * *=F D R<0.001$. (B) APOBEC3G expression in isolated CD4+ T cells from HIV-infected ART-suppressed individuals, treated as described in panel A. Statistical comparisons were performed using two-tailed Wilcoxon signed-rank tests compared to the DMSO-treated control. (C-D) APOBEC3G protein expression in CD4+ T cells treated with either $500 \mathrm{nM}$ rGal-9 or interferon- $\alpha$ (5000 units $/ \mathrm{ml})$, as determined by western blot. Immunoblotting bands were quantified with ImageJ software. The quantified APOBEC3G protein expression levels were normalized to corresponding Tubulin protein levels to account for variation in loading.

doi:10.1371/journal.ppat.1005677.g007

fluorescent protein (GFP) as an indicator of viral reactivation [34,35]. We performed doseresponse experiments by stimulating J-Lat $5 \mathrm{~A} 8,6.3$, and 11.1 clones with varying concentrations of rGal-9 for 24 hours. We used beads coated with anti-human $\alpha \mathrm{CD} 3(10 \mu \mathrm{g} / \mathrm{ml}$, eBiosciences, clone:OKT3) and $\alpha \mathrm{CD} 28(5 \mu \mathrm{g} / \mathrm{ml}$, eBiosciences, clone: CD28.2) to stimulate T cells in a manner that partially mimics stimulation by antigen-presenting cells as positive control. We used PMA/ionomycin $(16 \mathrm{nM} / 500 \mathrm{nM})$, and TNF $\alpha(10 \mathrm{ng} / \mathrm{ml})$ as positive controls. J-Lat cells were also stimulated with varying concentrations of galectins-1, 3, 4, 7, 8 to evaluate the ability of other recombinant galectins to reverse HIV latency. Flow cytometric analysis in a LSR II flow cytometer using the FACSDiva software (Becton Dickinson, Mountain View, CA) was used to assess mean fluorescence intensity of HIV-encoded GFP expression after stimulation. Data were analyzed with FlowJo (TreeStar Inc., Ashland, OR).

\section{Measurement of HIV latency reversal ex vivo}

Isolated CD4+ T cells were plated at a density of $1 \times 10^{6}$ cells per well at a volume of $1 \mathrm{~mL}$ Roswell Park Memorial Institute medium (RPMI) containing 20\% FBS (R20) in a 6-well flat-bottom plate. The cells were either untreated or treated with DMSO $0.5 \%$ as negative control, PMA (Sigma) at $2 \mathrm{nM}$ and Ionomycin (Sigma) at $0.5 \mu \mathrm{M}$, vorinostat at $1 \mu \mathrm{M}$, and varying concentrations of rGal-9 $(500 \mathrm{nM}$, and $1000 \mathrm{nM})$ for 24 hours. All stimuli solutions were prepared in R20 culture medium or DMSO solvent from stock solutions. The exposure time of cells to compounds was 24 hours. Total RNA was extracted using the AllPrep DNA/RNA/miRNA Universal Kit (Qiagen) with the optional on-column DNase treatment step. Cellular total HIV1 RNA was quantified with a qPCR TaqMan assay using LTR-specific primers F522-43 (5' GCC TCA ATA AAG CTT GCC TTG A 3'; HXB2 522-543) and R626-43 (5' GGG CGC CAC TGC TAG AGA 3'; 626-643) coupled with a FAM-BQ probe (5' CCA GAG TCA CAC AAC AGA CGG GCA CA 3) on a ViiA7 Real-time PCR System (Applied Biosystems, Inc. Foster City, CA, USA). Cellular total HIV-1 RNA copy number was determined in a reaction volume of $20 \mu \mathrm{l}$ with $10 \mu \mathrm{l}$ of $2 \mathrm{x}$ TaqMan RNA-to-Ct 1 Step kit (Life Technologies), 4 pmol of each primer, $4 \mathrm{pmol}$ of probe, $0.5 \mu \mathrm{l}$ reverse transcriptase, and $5 \mu \mathrm{l}$ of RNA. Cycling conditions were $48^{\circ} \mathrm{C}$ for $20 \mathrm{~min}, 95^{\circ} \mathrm{C}$ for $10 \mathrm{~min}$, then $60 \mathrm{cycles}$ of $95^{\circ} \mathrm{C}$ for $15 \mathrm{sec}$ and $59^{\circ} \mathrm{C}$ for $1 \mathrm{~min}$. External quantitation standards were prepared from full length NL4-3 virion RNA. Specimens were assayed with up to $500 \mathrm{ng}$ cellular total RNA in replicate reaction wells and copy number was determined by extrapolation against a 7-point standard curve (1-10,000 copies) performed in triplicate. Nucleic acid input was normalized for cell number using $18 \mathrm{~S}$ housekeeping gene copy number by qPCR. For cellular 18S RNA qPCR, the reaction volume was $20 \mu \mathrm{l}$ with $10 \mu \mathrm{l}$ of 2x TaqMan RNA to Ct 1 Step kit, $1 \mu$ of human 18 S Endogenous Control (Life technologies), $0.5 \mu \mathrm{l}$ reverse transcriptase, and $5 \mu \mathrm{l}$ of RNA. Cycling conditions were $48^{\circ} \mathrm{C}$ for $20 \mathrm{~min}$, $95^{\circ} \mathrm{C}$ for $10 \mathrm{~min}$, then $40 \mathrm{cycles}$ of $95^{\circ} \mathrm{C}$ for $15 \mathrm{sec}$ and $60^{\circ} \mathrm{C}$ for $1 \mathrm{~min} .18 \mathrm{~S}$ external standard curves ( 8 points curve, from 1-100,000 copies) were prepared from RNA extracted from HIV1-uninfected $\mathrm{CD}^{+} \mathrm{T}$ cells. 
A

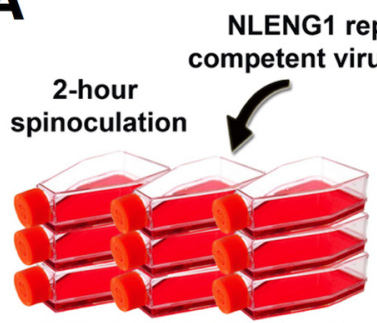

MOLT4-CCR5 cells 5 million ( $1 \times 10^{6}$ cells per ml R10)

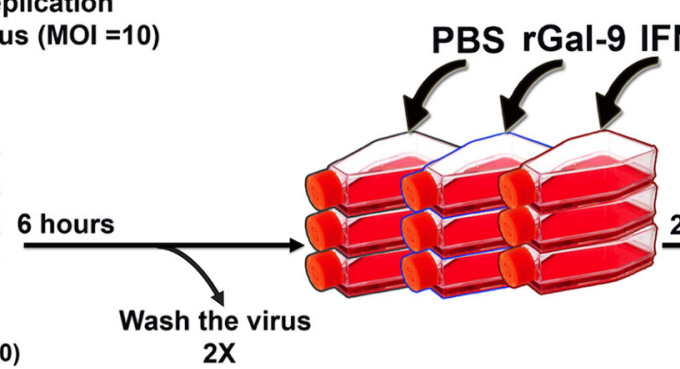

$2 X$
24 hours

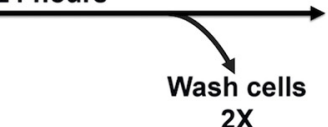

$2 \mathrm{X}$
Integrated HIV DNA
quantification

by $\mathrm{qPCR}$ at days $3,6,9,12$

Post-infection of target cells

(Target cells HIV infection)

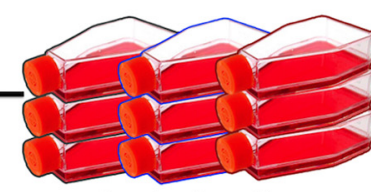

Jurkat cells

5 million ( $1 \times 10^{6}$ cells per $\left.\mathrm{ml} \mathrm{R} 10\right)$

\section{Concentrated culture supernatants \\ 2-hour spinoculation $\downarrow$}

Aliquot of concentrated culture supernatants

\section{HIV p24 quantification}

by ELISA

(producer cells HIV production)

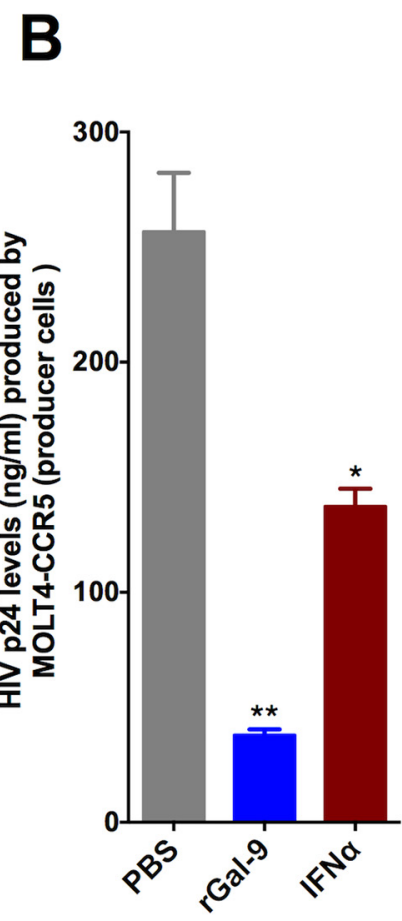

\section{C}

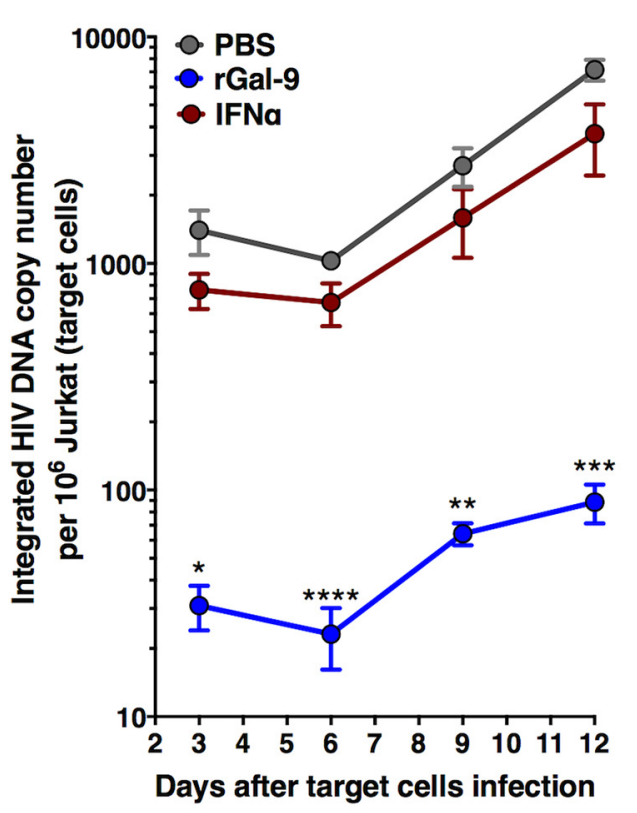

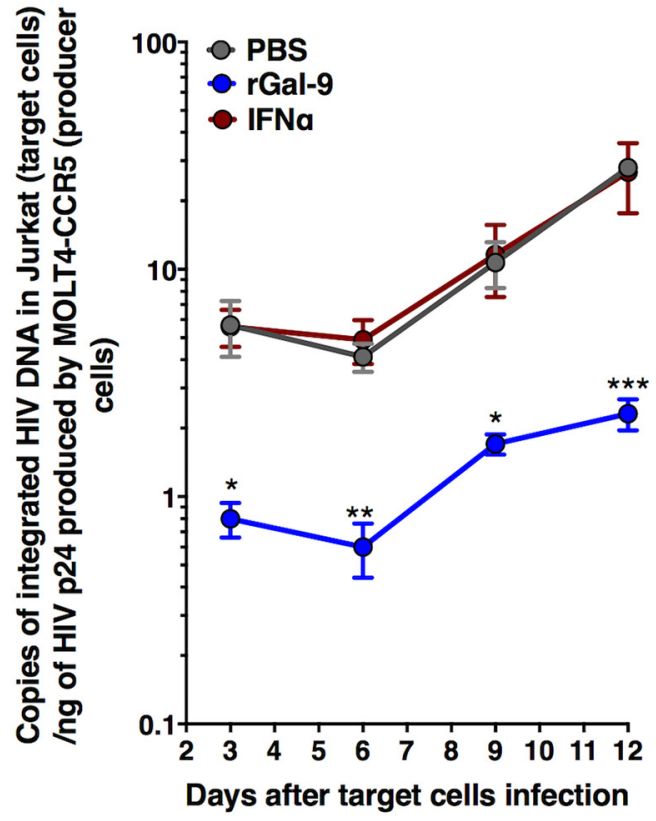

Fig 8. rGal-9 treatment reduces viral infectivity. (A) Illustrative schematic of the viral infectivity experiment. (B-D) Effects of rGal- 9 treatment of producer cells on HIV infectivity. The MOLT4-CCR5 cell line was infected for 6 hours, cells were washed and treated with either PBS, rGal-9 200nM, or interferon- $\alpha(5000 \mathrm{U} / \mathrm{ml})$ for 24 hours, and cultures were incubated for 3 days. (B) HIV p24 levels produced by MOLT4-CCR5 cells were quantified after concentrating the culture supernatants. Concentrated culture supernatants were used to infect Jurkat cells by spinoculation. (C) Levels of integrated HIV DNA measured at days $3,6,9$, and 12 post-infection of Jurkat cells. (D) Levels of integrated HIV DNA at days 3, 6, 9 , and 12 post- 
infection of Jurkat cells, normalized to producer cell p24 supernatant levels. Mean \pm SEM is displayed, and statistical comparisons were performed using two-tailed unpaired t test. ${ }^{*}=p<0.05 ;{ }^{*}=p<0.01,{ }^{* *}=p<0.001$, and ${ }^{* * * *}=p<0.0001$.

doi:10.1371/journal.ppat.1005677.g008

\section{Depletion of CD4+ T cells harboring CD69, CD25, and HLA-DR activation markers}

CD4+ T cells were isolated from PBMCs of three HIV-infected ART-suppressed individuals using negative selection (EasySep, STEMCELL Technologies). Resting CD4+ T cells were further enriched through depletion of cells expressing CD69, CD25, or HLA-DR surface markers from half of the isolated CD4+ T cells (CD69 MicroBead Kit II, Miltenyi Biotec; CD25

MicroBeads, Miltenyi Biotec; Anti-HLA-DR MicroBeads, Miltenyi Biotec). The remaining half was processed through the exact enrichment produce, except PBS was added instead of the depleting antibodies. Both of these cell populations were treated with 0.5\% DMSO (negative control), $500 \mathrm{nM}$ rGal-9, $1000 \mathrm{nM}$ rGal-9 or $\alpha \mathrm{CD} 3 / \alpha \mathrm{CD} 28$-conjugated beads. Induction of cell-associated HIV RNA was measured 24 hours post treatment using RT-qPCR.

\section{Quantitative analysis of synergy of latency reversing agent combinations}

CD4+ T cells isolated from five HIV-infected ART-suppressed individuals were treated with $500 \mathrm{nM}$ of rGal-9, $1 \mu \mathrm{M}$ vorinostat, $40 \mathrm{nM}$ romidepsin, $10 \mathrm{nM}$ bryostatin, $300 \mathrm{nM}$ prostratin, $1 \mu \mathrm{M}$ JQ1, or $30 \mathrm{nM}$ panobinostat alone or in combination with $500 \mathrm{nM}$ of rGal-9, in addition to $\alpha \mathrm{CD} 3+\alpha \mathrm{CD} 28$-conjugated beads (Dynal, at 1:1 bead:cell ratio) as a positive control. Fold induction of cell-associated HIV RNA was determined using quantitative real-time PCR 24 hours after treatment. qPCR data were normalized using three independent methods; cell count post-treatment and immediately prior to total RNA extraction, $18 \mathrm{~S}$ housekeeping gene quantification by qPCR, and RNA mass quantity measured by NanoDrop Spectrophotometer ND-1000 (NanoDrop Technologies). We adapted the Bliss independence model [48] as implemented by Jiang et al and Laird et al $[14,43]$ to test for synergy when rGal-9 500nM was combined with several latency reversing agents ex vivo. For drugs $\mathrm{x}$ and $\mathrm{y}$, we used the equation

A

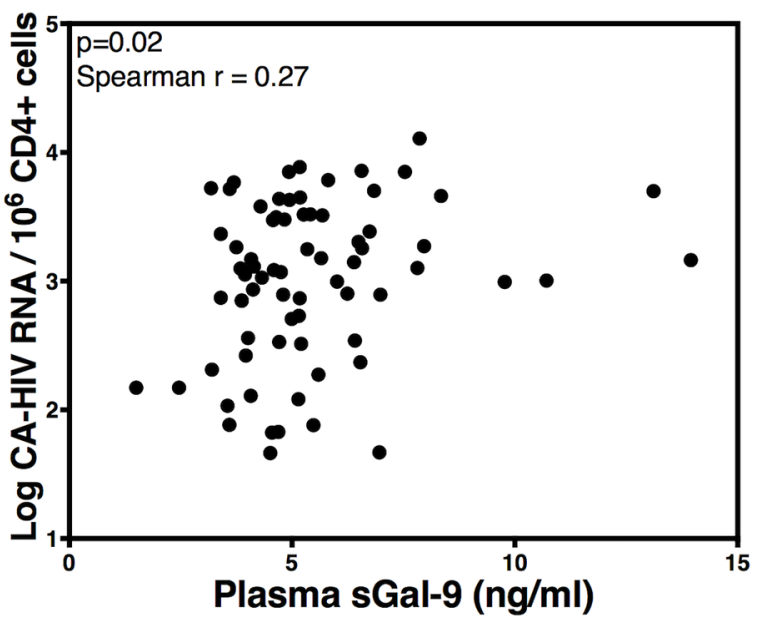

B

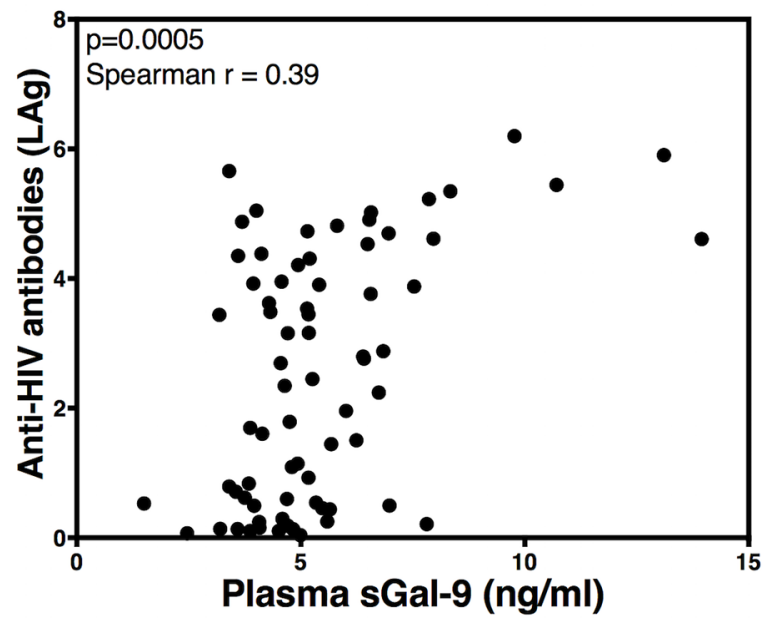

Fig 9. sGal-9 levels correlate with measures of HIV transcription and viral production in vivo. Correlations between levels of soluble Gal-9 and (A) levels of HIV cell-associated RNA, and (B) anti-HIV-1 antibodies in the plasma of 72 HIV-infected ARTsuppressed individuals. Correlations were evaluated using Spearman's rank correlation coefficient tests.

doi:10.1371/journal.ppat.1005677.g009 
$\mathrm{fa}_{\mathrm{xyP}}=\mathrm{fa}_{\mathrm{x}}+\mathrm{fa}_{\mathrm{y}}-\left(\mathrm{fa}_{\mathrm{x}}\right)\left(\mathrm{fa}_{\mathrm{y}}\right)$, where $\mathrm{fa}_{\mathrm{xy}}$ represents the predicted fraction affected by the combination of drug $\mathrm{x}$ and drug $\mathrm{y}$ given the observed effects of drug $\mathrm{x}\left(\mathrm{fa}_{\mathrm{x}}\right)$ and drug $\mathrm{y}\left(\mathrm{fa}_{\mathrm{y}}\right)$ administered individually and $\mathrm{fa}_{\mathrm{xy}}, \mathrm{O}=$ the observed effect when $\mathrm{x}$ and $\mathrm{y}$ were tested together. Calculation of $\mathrm{fa}_{\mathrm{x}}$ utilized the following approach adapted from the above cited publications: HIV RNA: $\mathrm{fa}_{\mathrm{x}}=$ (HIV RNA copies with drug $\mathrm{x}$-background copies with DMSO)/ (HIV RNA copies with $\alpha \mathrm{CD} 3-\alpha \mathrm{CD} 28$ stimulation-background copies with DMSO). In cases where one or more experimental drug conditions resulted in RNA expression exceeding the $\alpha \mathrm{CD} 3$ $\alpha \mathrm{CD} 28$ stimulation, we imputed the highest HIV RNA value in that experiment +1 to represent the denominator for calculation of $\mathrm{fa}_{\mathrm{x}} \cdot \Delta \mathrm{fa}_{\mathrm{xy}}=\mathrm{fa}_{\mathrm{xyO}}$ (the observed fraction affected by the drug combination) - $\mathrm{fa}_{\mathrm{xyP}}$ (the predicted fraction affected by the drug combination) provides an indication of synergy $\left(\Delta \mathrm{fa}_{\mathrm{xy}}>0\right)$, additive effect (Bliss independence) $\left(\Delta \mathrm{fa}_{\mathrm{xy}}=0\right)$, or antago$\operatorname{nism}\left(\Delta \mathrm{fa}_{\mathrm{xy}}<0\right)$. Statistical significance was determined using two-tailed paired $\mathrm{t}$-tests.

\section{Blocking of Tim-3, PDI, or CD44 receptors}

J-Lat 5A8 cells were either untreated were treated with one of the following: $30 \mathrm{mM}$ of $\alpha$-Lactose monohydrate (Sigma), $5 \mu \mathrm{g} / \mathrm{ml}$ rat anti-human TIM-3 monoclonal antibody (R\&D systems, Clone \# 344801), $5 \mu \mathrm{g} / \mathrm{ml}$ mouse anti-human PDI monoclonal antibody (Millipore, Clone \# 1D3), or $5 \mu \mathrm{g} / \mathrm{ml}$ rat anti-human CD44 monoclonal antibody (Thermo-scientific, Clone \# Hermes-1). After 30 minutes of incubation, cells were treated with $200 \mathrm{nM}$ of rGal-9. $\alpha \mathrm{CD} 3(10 \mu \mathrm{g} / \mathrm{ml})$ and $\alpha \mathrm{CD} 28(5 \mu \mathrm{g} / \mathrm{ml})$ stimulation was used as a positive control. Glucose and galactose $(30 \mathrm{mM})$ (Sigma) were used to examine the effects of other saccharide derivatives on rGal-9-mediated HIV latency reversal. Mean fluorescence intensity of HIV-encoded GFP expression in the $5 \mathrm{~A} 8$ cells was assessed using flow cytometry after 24 hours.

\section{Cell deglycosylation assay}

$\mathrm{J}$-Lat $5 \mathrm{~A} 8$ cells were either left untreated, or treated with $1 \mu \mathrm{g} / \mathrm{mL}$ tunicamycin (Sigma, St. Louis, MO), enzymatic protein deglycosylation mix (Sigma, St. Louis, MO), or individual deglycosylation enzymes for 24 hours. Cells were washed with RPMI media with $20 \%$ FBS, then were either left unstimulated or stimulated with rGal-9 (200 nM), PMA/inonmycin (16 $\mathrm{nM}, 500 \mathrm{nM})$, or TNF $\alpha(10 \mathrm{ng} / \mathrm{ml})$ for $12 \mathrm{hrs}, 24 \mathrm{hrs}$, or $48 \mathrm{hrs}$. Mean fluorescence intensity of HIV-encoded GFP expression in the 5A8 cells was assessed using flow cytometry after 24 hours.

\section{RNA-sequencing of CD4+ T cell transcriptome}

J-Lat 5A8 cells were either untreated, or treated with $200 \mathrm{nM}$ of rGal-9, $\alpha \mathrm{CD} 3(10 \mu \mathrm{g} / \mathrm{ml})$ and $\alpha \mathrm{CD} 28(5 \mu \mathrm{g} / \mathrm{ml})$, or a combination of $200 \mathrm{nM}$ rGal- $9+\alpha \mathrm{CD} 3$ and $\alpha \mathrm{CD} 28$ for 24 hours. Cells were sorted based on their GFP expression using the BD FACSAria III. Total RNA was extracted using the AllPrep DNA/RNA/miRNA Universal Kit (Qiagen) with the optional oncolumn DNase treatment step. RNA was quantified using a NanoDrop Spectrophotometer ND-1000 (NanoDrop Technologies) and integrity was assessed using a 2100 Bioanalyzer (Agilent Technologies). cDNAs were generated using the Illumina TruSeq Stranded mRNA Sample Preparation kit (Illumina Technologies) using $400 \mathrm{ng}$ of total RNA as input. Paired-end sequencing was performed using the Illumina HiSeq 2000 instrument to obtain 30-50 million $2 \times 51$ bp reads. RNA-Seq data were preprocessed by adaptor trimming and low quality 3 -tail trimming (Phred $>20$ ). The preprocessed reads were mapped using Tophat[72] to the reference genome hg19. Gene level expression quantification in FPKM (Fragments Per Kilobase of transcript per Million mapped reads) was calculated using Cufflinks suite including Cufflinks, Cuffmerge, Cuffquant and Cuffnorm[72]. Significant changes in transcript expression were 
quantified using a fold change cutoff ( $>2$ fold) and $t$ tests to determine significance, adjusted for false discovery rate (FDR $<0.05$ ). The gene annotations and gene ontology terms were extracted from BioMart using the Bioconductor/biomaRt package [72].

\section{CD4+ $T$ cell activation assay}

The surface expression of CD69 and CD25 markers of T-cell activation were measured using flow cytometry. Isolated CD4+ T cells were either treated with DMSO $0.5 \%$ as negative control, PMA $(2 \mathrm{nM})$ and Ionomycin $(0.5 \mathrm{mM})$, vorinostat $(1 \mu \mathrm{g} / \mathrm{ml})$, or two concentrations of rGal-9 $(500 \mathrm{nM}$, and $1 \mu \mathrm{M})$ for 24 hours. Cells were stained with LIVE/DEAD Fixable Aqua Dead Cell Stain Kit (Invitrogen) and then stained with the following fluorescently-conjugated monoclonal antibodies: V450 mouse anti-human CD69 (BD Biosciences, clone: FN50) and APC-cy7 mouse anti-human CD25 (BD Biosciences, clone: M-A251). Rainbow beads (Spherotec) were used to standardize instrument settings between runs. Data were analyzed using FlowJo (TreeStar Inc., Ashland, OR).

\section{Proliferation assays}

CD4+ T cells were negatively selected from thawed cryopreserved PBMCs. Isolated CD4+ T cells were stained with carboxyfluorescein diacetate succinimidyl ester (CFSE) at a final concentration of $10 \mu \mathrm{M}$ for $15 \mathrm{~min} .1$ X $10^{6} \mathrm{CFSE}$-stained CD4+ T cells were either left unstimulated, or stimulated for 24 hours with rGal-9 (200nM, and 500nM). After 24 hours, cells were washed and cultured for another 4 days. Cells were stained with LIVE/DEAD Fixable Aqua Dead Cell Stain Kit (Invitrogen) and then stained with the following fluorescently-conjugated monoclonal antibodies: Brilliant violet 421 anti-human CD4 (BioLegend, clone: OKT4) and FE/cy7 antihuman CD45RA (BioLegend, clone: HI100). Rainbow beads (Spherotec) were used to standardize instrument settings between runs. Data were acquired on the flow cytometer as above and were analyzed with FlowJo (TreeStar Inc., Ashland, OR).

\section{Quantitative PCR gene expression profiling}

400ng of RNA were transcribed into cDNA using random primers and the SuperScript VILO cDNA Synthesis Kit (Invitrogen), according to manufacturer's instructions. Quantitative realtime PCR utilized custom-made TaqMan Low Density Arrays (TLDA) from Applied Biosystems following the manufacturer's instructions. Thermal cycling was performed using an Applied Biosystems ViiA 7 Real-Time PCR System. Up to $450 \mathrm{ng}$ cDNA in $200 \mu \mathrm{l}$ of Applied Biosystems TaqMan Universal PCR Master Mix with UNG was loaded onto the designated ports of the TLDA plates. Data was analyzed using the Applied Biosystems ViiA 7 software. A panel of 6 housekeeping genes was included in the TLDA plates (GAPDH, 18S, ACTB, PPIA, RPLP0, and UBC). 18S ribosomal RNA was identified as the most stably expressed gene from this panel among all samples using the GeNorm algorithm[73]. Therefore, raw cycle threshold numbers of amplified gene products were normalized to $18 \mathrm{~S}$ ribosomal RNA to control for cDNA input amounts. Fold induction was determined using the comparative Ct method [73].

\section{Droplet digital PCR gene expression profiling}

Starting with 10 ng of input RNA, absolute quantification of HIV gag, p21 (Life technologies), and APOBEC3G (Life technologies) mRNA was performed, in addition to RNAse P (Ribonuclease $\mathrm{P}$ ) as a housekeeping gene, in four-plex digital droplet PCR reactions using the RainDrop system (RainDance Technologies). The RainDrop Source instrument was used with a microfluidic chip (RainDrop Source chip) containing 8 sample wells to generate a collection of 
uniformly sized (5 picoliter) aqueous droplets from each sample mixed with assay reagents in a reaction volume of $25 \mu \mathrm{l}$ with $12.5 \mu \mathrm{l}$ of SuperScript III, $1 \mu \mathrm{L}$ of SuperScript III Taq Polymerase (Life Technologies), $2.5 \mu \mathrm{L}$ 10x Droplet Stabilizer (RainDance Technologies), $2.5 \mu$ l of each primer/probe mixes, and $5 \mu \mathrm{l}$ of RNA. Droplets were thermocycled at $50^{\circ} \mathrm{C}$ for $25 \mathrm{~min}, 95^{\circ} \mathrm{C}$ for $10 \mathrm{~min}$, then underwent $45 \mathrm{cycles}$ of $95^{\circ} \mathrm{C}$ for $15 \mathrm{sec}$ and $60^{\circ} \mathrm{C}$ for $1 \mathrm{~min}$. The thermalcycled 8-tube strip was placed into the deck of the RainDrop Sense instrument with a second microfluidic chip (RainDrop Sense chip) used for single droplet fluorescence measurements. Data were analyzed using the RainDrop Analyst software. Data from each sample or control was converted to a 2-dimensional histogram displaying FAM intensity on the $\mathrm{x}$-axis and VIC intensity on the y-axis. Spectral compensation factors were calculated from the positive control data and applied to all samples. Gates used to count the number of droplet events with specific fluorescence properties were defined using graphical tools to outline regions from the positive control, and these gates were applied to all samples. For each sample, the number of PCR-positive droplet events was counted within each gate. Droplet counts were normalized to RNAseP counts.

\section{Western blotting}

Cells were lysed in radioimmunoprecipitation assay buffer $(150 \mathrm{~mm} \mathrm{NaCl}, 1 \%$ Nonidet P-40 (vol/vol), 0.5\% AB-deoxycholate (vol/vol), 0.1\% sodium dodecyl sulfate (SDS) (vol/vol), 50 $\mathrm{mm}$ Tris-HCl (pH 8), $1 \mathrm{~mm}$ DTT), and EDTA-free Protease Inhibitor (Calbiochem). Cellular lysates were used for SDS-polyacrylamide gel electrophoresis (SDS-PAGE) immunoblotting analysis. The primary antibodies used were anti-APOBEC3G mAb (NIH AIDS Reagent Program, Division of AIDS, NIAID, NIH: Anti-APOBEC3G from Dr. Warner C. Greene [74]), anti-p24 mAb (NIH AIDS Reagent Program, Division of AIDS, NIAID, NIH: Anti-HIV-1 p24 Monoclonal (71-31) from Dr. Susan Zolla-Pazner [75]), anti-Vif mAb (NIH AIDS Reagent Program, Division of AIDS, NIAID, NIH: HIV-1 Vif Monoclonal Antibody (\#319)) from Dr. Michael H. Malim [76-78]), and anti-Tubulin (B-Tubulin 926-42211 from LI-COR). The immunoblotting bands were quantified using ImageJ software.

\section{Infectivity assay and virion quantification by p24 ELISA}

Five million MOLT4-CCR5 cells ( $1 \times 10^{6}$ cells per $\mathrm{ml}$ of R10 media) were spinoculated with NLENG1 replication competent virus $(\mathrm{MOI}=10)$ for 2 hours, cells were incubated for 6 hours at $37^{\circ} \mathrm{C}$, then washed twice and were treated with PBS, $200 \mathrm{nM} \mathrm{rGal}-9$, or IFN $\alpha(5000 \mathrm{U} / \mathrm{ml})$ for 24 hours. Then, cells were washed twice and left in culture for 3 additional days. Culture supernatants were concentrated using Lenti-X Concentrator (Clontech) and aliquots of the concentrated culture supernatants were used to measure HIV production through the quantification of viral p24 antigen by p24 HIV Antigen ELISA (PerkinElmer), according to the manufacturer's instructions. The remaining equal amounts of concentrated culture supernatants were used to infect 5 million Jurkat cells ( $1 \times 10^{6}$ cells per $\mathrm{ml}$ of R10 media) by spinoculation for two hours. Jurkat cells were collected at days 3, 6, 9, and 12 post-infection, and total genomic DNA was extracted using the AllPrep DNA/RNA/miRNA Universal Kit (Qiagen). qPCR was used to quantify the levels of integrated HIV-1 as described below [79].

\section{Quantification of integrated HIV DNA by qPCR}

Integrated HIV DNA levels were quantified using two-step PCR reactions, as previously described[79]. Integrated HIV-1 DNA was pre-amplified with two Alu primers and a primer specific for the HIV LTR region, in addition to primers specific for the CD3 gene to determine cell count. Nested qPCR was then used to amplify HIV and CD3 sequences from the first 
round of amplification. Specimens were assayed with up to $500 \mathrm{ng}$ cellular DNA in triplicate reaction wells and copy number was determined by extrapolation against a 5-point standard curve (3-30,000 copies), performed in triplicate using extracted DNA from ACH-2 cells.

\section{sGal-9 level measurement by ELISA}

The levels of sGal-9 in the plasma of 72 ART-suppressed HIV-infected individuals were measured using the solid-phase Human Galectin-9 Quantikine ELISA Kit (R\&D systems) according to the manufacturer's instructions.

\section{Measurement of anti-HIV antibodies in plasma}

The Limiting Antigen-Avidity (LAg) EIA (Sedia Biosciences, Portland, OR) was used to quantify and characterize the HIV-specific antibody profile in $72 \mathrm{HIV}$-infected individuals on suppressive ART. The single-well plate-based LAg EIA measures the quantity and avidity of HIVspecific IgG to subtype B, E and D recombinant HIV-1 envelope antigens $[4,54,55]$.

\section{Statistical analysis}

The nonparametric Mann-Whitney U test was used for unpaired comparisons, Wilcoxon matched-pairs signed ranked test was used for paired comparisons, and Spearman's $r$ and Pearson's $r$ tests were used for correlation analyses (the Shapiro-Wilk test was used to determine normality prior to implementation of the Pearson's $r$ test). All statistical analyses were conducted using GraphPad Prism release 6.0 (GraphPad Software, San Diego, CA, USA) and statistical significance was set at a p-value of 0.05 . False discovery rates (FDR) were computed using the Benjamini-Hochberg procedure [80] to adjust for multiple comparisons in the RNAseq data and qPCR array analysis. The heatmaps were generated using standardized Z-scores, and the clustering dendrogram depicting relatedness between gene expression profiles was generated using hierarchical clustering with complete linkage (created using the R statistical package).

\section{Supporting Information}

S1 Fig. rGal-9 reactivates latent HIV in vitro in an exposure time-dependent fashion. Effects of $1000 \mathrm{nM} \mathrm{rGal}-9$ pulse treatment for 1 hour, 2 hours, 4 hours, and 6 hours compared to continuous treatment for 24 hours. J-Lat cells were analyzed by flow cytometry after 24 hours of culture to assess HIV-encoded GFP expression. Mean \pm SEM is displayed, and statistical comparisons were performed using two-tailed unpaired $\mathrm{t}$ test. ${ }^{*}=\mathrm{p}<0.05{ }^{* *}=\mathrm{p}<0.01,{ }^{* * *}=$ $\mathrm{p}<0.001$ and $^{* * * *}=\mathrm{p}<0.0001$.

S2 Fig. rGal-9 treatment induces the expression of HIV proteins in vitro. (A) HIV p24 and Vif protein expression in J-Lat 5A8 cells treated with varying concentrations of rGal-9 (100 $\mathrm{nM}, 200 \mathrm{nM}$, and $500 \mathrm{nM})$ or interferon- $\alpha$ (5000 units $/ \mathrm{ml}$ ), as determined by western blot.

(B-C) Immunoblotting bands were quantified with ImageJ software. The quantified HIV p24

(B) and Vif (C) protein expression levels were normalized to corresponding Tubulin protein levels to account for variation in loading.

(TIFF)

S3 Fig. Limited-term exposure to rGal-9 reactivates latent HIV ex vivo in CD69 / CD25 / HLA-DR-depleted CD4+ T cells. Effects of $500 \mathrm{nM}$ or $1000 \mathrm{nM}$ rGal-9 treatment for 6 hours compared to continuous treatment for 24 hours. CD4+ T cells were isolated from PBMCs of 
three HIV-infected ART-suppressed individuals using negative selection. Resting CD4+ T cells were further enriched through depletion of cells expressing CD69, CD25, or HLA-DR surface markers from half of the isolated CD4+ T cells. Cells were treated with $0.5 \%$ DMSO (negative control), $500 \mathrm{nM}$ rGal-9, $1000 \mathrm{nM} \mathrm{rGal}-9$ or $\alpha \mathrm{CD} 3 / \alpha \mathrm{CD} 28$-conjugated beads for either 6 hours or 24 hours. Induction of cell-associated HIV RNA was measured 24 hours post treatment using RT-qPCR. Mean \pm SEM is displayed. Percentages reported reflect average values measured in the 6 hours treatment with respect to values observed with continuous treatment for 24 hours at the same concentration. Each individual is represented with a different symbol. (TIFF)

S4 Fig. Effects of rGal-9 administration on cell viability ex vivo. Percentage of live CD4+ $\mathrm{T}$ cells from three ART-suppressed individuals treated with $0.5 \%$ DMSO as negative control, 500 $\mathrm{nM}$ rGal-9, or $1000 \mathrm{nM}$ rGal-9 for 24 hours. LIVE/DEAD Fixable Aqua Dead Cell Staining was used to assess the cellular viability. Mean \pm SEM is displayed.

S5 Fig. Glucose and galactose do not inhibit rGal-9-mediated latent viral reactivation. Effects of $\alpha$ Lactose $(30 \mathrm{mM})$, glucose $(30 \mathrm{mM})$, and galactose $(30 \mathrm{mM})$ treatment on rGal9-mediated reactivation of HIV in J-Lat $5 \mathrm{~A} 8$ cells. J-Lat cells were analyzed by flow cytometry to assess HIV-encoded GFP expression.

S6 Fig. Reactivation of latent HIV by PMA/Ionomycin and TNF $\alpha$ is not reduced by deglycosylation. (A), Effects of $1 \mu \mathrm{g} / \mathrm{ml}$ tunicamycin treatment on the ability of PMA/Ionomycin and TNF $\alpha$ to reactivate latent HIV in J-Lat 5A8 cells. (B) Effects of deglycosylation enzymatic mix treatment on the ability of PMA/Ionomycin and TNF $\alpha$ to reactivate latent HIV in J-Lat 5 A8 cells. Mean \pm SEM is displayed.

(TIFF)

S7 Fig. rGal-9 treatment induces a unique gene expression signature in J-Lat cells. Cluster dendrogram depicting relatedness between individual gene expression profiles of sorted GFPpositive and GFP-negative cells containing reactivated (transcriptionally active) HIV proviruses and latent (transcriptionally inactive) proviruses, respectively, after rGal-9 stimulation, $\alpha \mathrm{CD} 3 / \alpha \mathrm{CD} 28$ stimulation, or a combination of both. The dendrogram was generated using hierarchical clustering with complete linkage.

(TIFF)

S8 Fig. rGal-9 modulates the expression of selected genes associated with $\mathrm{T}$ cell activation, T cell proliferation and $T$ cell apoptosis. Heat maps demonstrate the effects of rGal-9 treatment on the expression of (A) T cell activation genes, (B) genes involved in positive regulation of T cell proliferation, $(\mathbf{C})$ genes involved in negative regulation of $\mathrm{T}$ cell proliferation, and (D) T cell apoptosis genes. Heat colors show standardized Z-scores across samples; red indicates upregulated expression, and green indicates downregulated expression. Heat maps only show genes modulated $>2$ fold with FDR $<0.05$. Gene names in red represent genes that were upregulated in the rGal-9-treated, GFP+ cells as compared to unstimulated control. Gene names in green represent genes that were downregulated in rGal-9-treated, GFP+ cells as compared to unstimulated control. The gene annotations and gene ontology terms were extracted from BioMart using the Bioconductor/biomaRt package.

(TIFF)

S9 Fig. rGal-9 modulates the expression of selected genes associated with innate immunity, cytokine production, and interferon signaling. Heat maps demonstrate the effects of rGal-9 
treatment on the expression of (A) innate immunity-associated genes, (B) cytokine genes, and (C) interferon genes. Heat colors show standardized Z-scores across samples; red indicates upregulated expression, and green indicates downregulated expression. Heat maps only show genes modulated $>2$ fold with $\mathrm{FDR}<0.05$. Gene names in red represent genes that were upregulated in the rGal-9-treated, GFP+ cells as compared to unstimulated control. Gene names in green represent genes that were downregulated in rGal-9-treated, GFP+ cells as compared to unstimulated control. The gene annotations and gene ontology terms were extracted from BioMart using the Bioconductor/biomaRt package.

(TIFF)

S10 Fig. rGal-9 treatment induces the expression of several anti-HIV host restriction factors including APOBEC3G in vitro. Heat map depicts expression levels of host restriction factors in sorted GFP-positive and GFP-negative J-Lat 5A8 cells containing reactivated (transcriptionally active) HIV proviruses and latent (transcriptionally inactive) proviruses, respectively, after rGal-9 stimulation, $\alpha \mathrm{CD} 3 / \alpha \mathrm{CD} 28$ stimulation, or a combination of both. Heat colors scale with fold modulation compared to the unstimulated control as described in the figure.

(TIFF)

S11 Fig. Modulation of gene expression by rGal-9 is similar in vitro and ex vivo. (A) Correlations between gene expression modulation of 42 anti-HIV-1 host restriction factors in J-Lat $5 \mathrm{~A} 8$ cells treated with $200 \mathrm{nM}$ of rGal-9, and primary CD4+ T cells from $10 \mathrm{HIV}$-infected, ART-suppressed individuals treated with either $500 \mathrm{nM}$ of rGal-9 or 1000nM of rGal-9. (B) Correlation between gene expression modulation of 42 anti-HIV-1 host restriction factors in primary CD4+ T cells treated with 500nM of rGal-9, and 1000nM of rGal-9. Correlations were evaluated using Pearson's $r$ tests after testing for normality using the Shapiro-Wilk test. (TIFF)

S1 Table. Subject characteristics.

(TIFF)

\section{Acknowledgments}

We would like to thank Paul Contestable and Ortho Clinical Diagnostics for providing the Vitros HIV-1+2 reagents.

\section{Author Contributions}

Conceived and designed the experiments: MAM LC RT GMC AD SK MLS LCN SKP. Performed the experiments: MAM LC RT GMC AD SK MLS LCN SKP. Analyzed the data: MAM XD. Contributed reagents/materials/analysis tools: ML JBS PJN TN MH LCN RH CMS SGD. Wrote the paper: MAM SKP.

\section{References}

1. Wong JK, Hezareh M, Gunthard HF, Havlir DV, Ignacio CC, et al. (1997) Recovery of replication-competent HIV despite prolonged suppression of plasma viremia. Science 278: 1291-1295. PMID: 9360926

2. Finzi D, Hermankova M, Pierson T, Carruth LM, Buck C, et al. (1997) Identification of a reservoir for HIV-1 in patients on highly active antiretroviral therapy. Science 278: 1295-1300. PMID: 9360927

3. Butler KM, Gavin P, Coughlan S, Rochford A, Mc Donagh S, et al. (2015) Rapid viral rebound after 4 years of suppressive therapy in a seronegative HIV-1 infected infant treated from birth. Pediatr Infect Dis J 34: e48-51. doi: 10.1097/INF.0000000000000570 PMID: 25742088 
4. Henrich TJ, Hanhauser E, Marty FM, Sirignano MN, Keating S, et al. (2014) Antiretroviral-free HIV-1 remission and viral rebound after allogeneic stem cell transplantation: report of 2 cases. Ann Intern Med 161: 319-327. doi: 10.7326/M14-1027 PMID: 25047577

5. Saez-Cirion A, Bacchus C, Hocqueloux L, Avettand-Fenoel V, Girault I, et al. (2013) Post-treatment HIV-1 controllers with a long-term virological remission after the interruption of early initiated antiretroviral therapy ANRS VISCONTI Study. PLoS Pathog 9: e1003211. doi: 10.1371/journal.ppat.1003211 PMID: 23516360

6. Siliciano JD, Siliciano RF (2013) HIV-1 eradication strategies: design and assessment. Curr Opin HIV AIDS 8: 318-325. doi: 10.1097/COH.0b013e328361eaca PMID: 23698561

7. Elliott JH, Wightman F, Solomon A, Ghneim K, Ahlers J, et al. (2014) Activation of HIV transcription with short-course vorinostat in HIV-infected patients on suppressive antiretroviral therapy. PLoS Pathog 10 e1004473. doi: 10.1371/journal.ppat.1004473 PMID: 25393648

8. Olesen R, Vigano S, Rasmussen T, Sogaard OS, Ouyang Z, et al. (2015) Innate immune activity correlates with CD4 T cell-associated HIV-1 DNA decline during latency-reversing treatment with panobinostat. J Virol.

9. Spivak AM, Andrade A, Eisele E, Hoh R, Bacchetti P, et al. (2014) A pilot study assessing the safety and latency-reversing activity of disulfiram in HIV-1-infected adults on antiretroviral therapy. Clin Infect Dis 58: 883-890. doi: 10.1093/cid/cit813 PMID: 24336828

10. Archin NM, Bateson R, Tripathy MK, Crooks AM, Yang KH, et al. (2014) HIV-1 expression within resting CD4+ T cells after multiple doses of vorinostat. J Infect Dis 210: 728-735. doi: 10.1093/infdis/jiu155 PMID: 24620025

11. Archin NM, Liberty AL, Kashuba AD, Choudhary SK, Kuruc JD, et al. (2012) Administration of vorinostat disrupts HIV-1 latency in patients on antiretroviral therapy. Nature 487: 482-485. doi: 10.1038/ nature11286 PMID: 22837004

12. Elliott JH, McMahon JH, Chang CC, Lee SA, Hartogensis W, et al. (2015) Short-term administration of disulfiram for reversal of latent HIV infection: a phase 2 dose-escalation study. Lancet HIV 2: e520529. doi: 10.1016/S2352-3018(15)00226-X PMID: 26614966

13. Sogaard OS, Graversen ME, Leth S, Olesen R, Brinkmann CR, et al. (2015) The Depsipeptide Romidepsin Reverses HIV-1 Latency In Vivo. PLoS Pathog 11: e1005142. doi: 10.1371/journal.ppat. 1005142 PMID: 26379282

14. Laird GM, Bullen CK, Rosenbloom DI, Martin AR, Hill AL, et al. (2015) Ex vivo analysis identifies effective HIV-1 latency-reversing drug combinations. J Clin Invest 125: 1901-1912. doi: 10.1172/JCl80142 PMID: 25822022

15. Harper JW, Adami GR, Wei N, Keyomarsi K, Elledge SJ (1993) The p21 Cdk-interacting protein Cip1 is a potent inhibitor of G1 cyclin-dependent kinases. Cell 75: 805-816. PMID: 8242751

16. Chen H, Li C, Huang J, Cung T, Seiss K, et al. (2011) CD4+ T cells from elite controllers resist HIV-1 infection by selective upregulation of p21. J Clin Invest 121: 1549-1560. doi: 10.1172/JCI44539 PMID: 21403397

17. Abdel-Mohsen M, Wang C, Strain MC, Lada SM, Deng X, et al. (2015) Select host restriction factors are associated with HIV persistence during antiretroviral therapy. AIDS 29: 411-420. doi: 10.1097/ QAD.0000000000000572 PMID: 25602681

18. Kurose $Y$, Wada J, Kanzaki M, Teshigawara S, Nakatsuka A, et al. (2013) Serum galectin-9 levels are elevated in the patients with type 2 diabetes and chronic kidney disease. BMC Nephrol 14: 23. doi: 10. 1186/1471-2369-14-23 PMID: 23339460

19. Baba M, Wada J, Eguchi J, Hashimoto I, Okada T, et al. (2005) Galectin-9 inhibits glomerular hypertrophy in db/db diabetic mice via cell-cycle-dependent mechanisms. J Am Soc Nephrol 16: 3222-3234. PMID: 16177004

20. Elahi S, Niki T, Hirashima M, Horton H (2012) Galectin-9 binding to Tim-3 renders activated human CD4+ T cells less susceptible to HIV-1 infection. Blood 119: 4192-4204. doi: 10.1182/blood-2011-11389585 PMID: 22438246

21. Barondes SH, Castronovo V, Cooper DN, Cummings RD, Drickamer K, et al. (1994) Galectins: a family of animal beta-galactoside-binding lectins. Cell 76: 597-598. PMID: 8124704

22. Kasai K, Hirabayashi J (1996) Galectins: a family of animal lectins that decipher glycocodes. J Biochem 119: 1-8. PMID: 8907168

23. Cooper DN (2002) Galectinomics: finding themes in complexity. Biochim Biophys Acta 1572: 209-231. PMID: 12223271

24. Sehrawat S, Reddy PB, Rajasagi N, Suryawanshi A, Hirashima M, et al. (2010) Galectin-9/TIM-3 interaction regulates virus-specific primary and memory CD8 T cell response. PLoS Pathog 6: e1000882. doi: 10.1371/journal.ppat.1000882 PMID: 20463811 
25. Tandon R, Chew GM, Byron MM, Borrow P, Niki T, et al. (2014) Galectin-9 is rapidly released during acute HIV-1 infection and remains sustained at high levels despite viral suppression even in elite controllers. AIDS Res Hum Retroviruses 30: 654-664. doi: 10.1089/AID.2014.0004 PMID: 24786365

26. Jost S, Moreno-Nieves UY, Garcia-Beltran WF, Rands K, Reardon J, et al. (2013) Dysregulated Tim-3 expression on natural killer cells is associated with increased Galectin-9 levels in HIV-1 infection. Retrovirology 10: 74. doi: 10.1186/1742-4690-10-74 PMID: 23866914

27. Wang F, He W, Yuan J, Wu K, Zhou H, et al. (2008) Activation of Tim-3-Galectin-9 pathway improves survival of fully allogeneic skin grafts. Transpl Immunol 19: 12-19. doi: 10.1016/j.trim.2008.01.008 PMID: 18346632

28. Seki M, Sakata KM, Oomizu S, Arikawa T, Sakata A, et al. (2007) Beneficial effect of galectin 9 on rheumatoid arthritis by induction of apoptosis of synovial fibroblasts. Arthritis Rheum 56: 3968-3976. PMID: 18050192

29. Katoh S, Ishii N, Nobumoto A, Takeshita K, Dai SY, et al. (2007) Galectin-9 inhibits CD44-hyaluronan interaction and suppresses a murine model of allergic asthma. Am J Respir Crit Care Med 176: 27-35. PMID: 17446336

30. Kuroda J, Yamamoto M, Nagoshi H, Kobayashi T, Sasaki N, et al. (2010) Targeting activating transcription factor 3 by Galectin- 9 induces apoptosis and overcomes various types of treatment resistance in chronic myelogenous leukemia. Mol Cancer Res 8: 994-1001. doi: 10.1158/1541-7786.MCR-10-0040 PMID: 20571063

31. Nobumoto A, Nagahara K, Oomizu S, Katoh S, Nishi N, et al. (2008) Galectin-9 suppresses tumor metastasis by blocking adhesion to endothelium and extracellular matrices. Glycobiology 18: 735744. doi: 10.1093/glycob/cwn062 PMID: 18579572

32. Gleason MK, Lenvik TR, McCullar V, Felices M, O'Brien MS, et al. (2012) Tim-3 is an inducible human natural killer cell receptor that enhances interferon gamma production in response to galectin-9. Blood 119: 3064-3072. doi: 10.1182/blood-2011-06-360321 PMID: 22323453

33. Mishra R, Grzybek M, Niki T, Hirashima M, Simons K (2010) Galectin- 9 trafficking regulates apicalbasal polarity in Madin-Darby canine kidney epithelial cells. Proc Natl Acad Sci U S A 107: 1763317638. doi: 10.1073/pnas.1012424107 PMID: 20861448

34. Chavez L, Kauder S, Verdin E (2011) In vivo, in vitro, and in silico analysis of methylation of the HIV-1 provirus. Methods 53: 47-53. doi: 10.1016/j.ymeth.2010.05.009 PMID: 20670606

35. Jordan A, Bisgrove D, Verdin E (2003) HIV reproducibly establishes a latent infection after acute infection of T cells in vitro. EMBO J 22: 1868-1877. PMID: 12682019

36. Chan JK, Bhattacharyya D, Lassen KG, Ruelas D, Greene WC (2013) Calcium/calcineurin synergizes with prostratin to promote NF-kappaB dependent activation of latent HIV. PLoS One 8: e77749. doi: 10.1371/journal.pone.0077749 PMID: 24204950

37. Wei DG, Chiang V, Fyne E, Balakrishnan M, Barnes T, et al. (2014) Histone deacetylase inhibitor romidepsin induces HIV expression in CD4 T cells from patients on suppressive antiretroviral therapy at concentrations achieved by clinical dosing. PLoS Pathog 10: e1004071. doi: 10.1371/journal.ppat. 1004071 PMID: 24722454

38. DeChristopher BA, Loy BA, Marsden MD, Schrier AJ, Zack JA, et al. (2012) Designed, synthetically accessible bryostatin analogues potently induce activation of latent HIV reservoirs in vitro. Nat Chem 4: 705-710. doi: 10.1038/nchem.1395 PMID: 22914190

39. Perez M, de Vinuesa AG, Sanchez-Duffhues G, Marquez N, Bellido ML, et al. (2010) Bryostatin-1 synergizes with histone deacetylase inhibitors to reactivate HIV-1 from latency. Curr HIV Res 8: 418429. PMID: 20636281

40. Kulkosky J, Culnan DM, Roman J, Dornadula G, Schnell M, et al. (2001) Prostratin: activation of latent HIV-1 expression suggests a potential inductive adjuvant therapy for HAART. Blood 98: 3006-3015. PMID: 11698284

41. Reuse S, Calao M, Kabeya K, Guiguen A, Gatot JS, et al. (2009) Synergistic activation of HIV-1 expression by deacetylase inhibitors and prostratin: implications for treatment of latent infection. PLoS One 4: e6093. doi: 10.1371/journal.pone.0006093 PMID: 19564922

42. Williams SA, Chen LF, Kwon H, Fenard D, Bisgrove D, et al. (2004) Prostratin antagonizes HIV latency by activating NF-kappaB. J Biol Chem 279: 42008-42017. PMID: 15284245

43. Jiang G, Mendes EA, Kaiser P, Wong DP, Tang Y, et al. (2015) Synergistic Reactivation of Latent HIV Expression by Ingenol-3-Angelate, PEP005, Targeted NF-kB Signaling in Combination with JQ1 Induced p-TEFb Activation. PLoS Pathog 11: e1005066. doi: 10.1371/journal.ppat.1005066 PMID: 26225771 
44. Li Z, Guo J, Wu Y, Zhou Q (2013) The BET bromodomain inhibitor JQ1 activates HIV latency through antagonizing Brd4 inhibition of Tat-transactivation. Nucleic Acids Res 41: 277-287. doi: 10.1093/nar/ gks976 PMID: 23087374

45. Zhu J, Gaiha GD, John SP, Pertel T, Chin CR, et al. (2012) Reactivation of latent HIV-1 by inhibition of BRD4. Cell Rep 2: 807-816. doi: 10.1016/j.celrep.2012.09.008 PMID: 23041316

46. Rasmussen TA, Tolstrup M, Brinkmann CR, Olesen R, Erikstrup C, et al. (2014) Panobinostat, a histone deacetylase inhibitor, for latent-virus reactivation in HIV-infected patients on suppressive antiretroviral therapy: a phase 1/2, single group, clinical trial. Lancet HIV 1: e13-21. doi: 10.1016/S2352-3018 (14)70014-1 PMID: 26423811

47. Spivak AM, Bosque A, Balch AH, Smyth D, Martins L, et al. (2015) Ex Vivo Bioactivity and HIV-1 Latency Reversal by Ingenol Dibenzoate and Panobinostat in Resting CD4(+) T Cells from Aviremic Patients. Antimicrob Agents Chemother 59: 5984-5991. doi: 10.1128/AAC.01077-15 PMID: 26169416

48. Bliss $\mathrm{Cl}$ (1939) The toxicity of poisons applied jointly Ann Appl Biol 26: 585-615. Find this article online.

49. Zhu C, Anderson AC, Schubart A, Xiong H, Imitola J, et al. (2005) The Tim-3 ligand galectin-9 negatively regulates Thelper type 1 immunity. Nat Immunol 6: 1245-1252. PMID: 16286920

50. Bi S, Hong PW, Lee B, Baum LG (2011) Galectin-9 binding to cell surface protein disulfide isomerase regulates the redox environment to enhance T-cell migration and HIV entry. Proc Natl Acad Sci U S A 108: 10650-10655. doi: 10.1073/pnas.1017954108 PMID: 21670307

51. Wu C, Thalhamer T, Franca RF, Xiao S, Wang C, et al. (2014) Galectin-9-CD44 interaction enhances stability and function of adaptive regulatory T cells. Immunity 41:270-282. doi: 10.1016/j.immuni. 2014.06.011 PMID: 25065622

52. Lecossier D, Bouchonnet F, Clavel F, Hance AJ (2003) Hypermutation of HIV-1 DNA in the absence of the Vif protein. Science 300: 1112. PMID: 12750511

53. Yu Q, Konig R, Pillai S, Chiles K, Kearney M, et al. (2004) Single-strand specificity of APOBEC3G accounts for minus-strand deamination of the HIV genome. Nat Struct Mol Biol 11: 435-442. PMID: 15098018

54. Keating SM, Hanson D, Lebedeva M, Laeyendecker O, Ali-Napo NL, et al. (2012) Lower-sensitivity and avidity modifications of the vitros anti-HIV $1+2$ assay for detection of recent HIV infections and incidence estimation. J Clin Microbiol 50: 3968-3976. doi: 10.1128/JCM.01454-12 PMID: 23035182

55. Yukl SA, Boritz E, Busch M, Bentsen C, Chun TW, et al. (2013) Challenges in detecting HIV persistence during potentially curative interventions: a study of the Berlin patient. PLoS Pathog 9: e1003347. doi: 10.1371/journal.ppat.1003347 PMID: 23671416

56. Lanteri M, Giordanengo V, Hiraoka N, Fuzibet JG, Auberger P, et al. (2003) Altered T cell surface glycosylation in HIV-1 infection results in increased susceptibility to galectin-1-induced cell death. Glycobiology 13: 909-918. PMID: 12925577

57. Ardman B, Sikorski MA, Settles M, Staunton DE (1990) Human immunodeficiency virus type 1-infected individuals make autoantibodies that bind to CD43 on normal thymic lymphocytes. J Exp Med 172: 1151-1158. PMID: 1976735

58. Giordanengo V, Limouse M, Desroys du Roure L, Cottalorda J, Doglio A, et al. (1995) Autoantibodies directed against CD43 molecules with an altered glycosylation status on human immunodeficiency virus type 1 (HIV-1)-infected CEM cells are found in all HIV-1+ individuals. Blood 86: 2302-2311. PMID: 7662977

59. Bi S, Earl LA, Jacobs L, Baum LG (2008) Structural features of galectin- 9 and galectin- 1 that determine distinct T cell death pathways. J Biol Chem 283: 12248-12258. doi: 10.1074/jbc.M800523200 PMID: 18258591

60. Pillai SK, Abdel-Mohsen M, Guatelli J, Skasko M, Monto A, et al. (2012) Role of retroviral restriction factors in the interferon-alpha-mediated suppression of HIV-1 in vivo. Proc Natl Acad Sci U S A 109: 3035-3040. doi: 10.1073/pnas.1111573109 PMID: 22315404

61. Sheehy AM, Gaddis NC, Malim MH (2003) The antiretroviral enzyme APOBEC3G is degraded by the proteasome in response to HIV-1 Vif. Nat Med 9: 1404-1407. PMID: 14528300

62. YukI SA, Shergill AK, McQuaid K, Gianella S, Lampiris H, et al. (2010) Effect of raltegravir-containing intensification on HIV burden and T-cell activation in multiple gut sites of HIV-positive adults on suppressive antiretroviral therapy. AIDS 24: 2451-2460. doi: 10.1097/QAD.0b013e32833ef7bb PMID: 20827162

63. Lucera MB, Tilton CA, Mao H, Dobrowolski C, Tabler CO, et al. (2014) The histone deacetylase inhibitor vorinostat (SAHA) increases the susceptibility of uninfected CD4+ T cells to HIV by increasing the kinetics and efficiency of postentry viral events. J Virol 88: 10803-10812. doi: 10.1128/JVI.00320-14 PMID: 25008921 
64. Nagahara K, Arikawa T, Oomizu S, Kontani K, Nobumoto A, et al. (2008) Galectin-9 increases Tim-3+ dendritic cells and CD8+ T cells and enhances antitumor immunity via galectin-9-Tim-3 interactions. $J$ Immunol 181: 7660-7669. PMID: 19017954

65. Chomont N, El-Far M, Ancuta P, Trautmann L, Procopio FA, et al. (2009) HIV reservoir size and persistence are driven by T cell survival and homeostatic proliferation. Nat Med 15: 893-900. doi: 10.1038/ nm.1972 PMID: 19543283

66. Seki M, Oomizu S, Sakata KM, Sakata A, Arikawa T, et al. (2008) Galectin-9 suppresses the generation of Th17, promotes the induction of regulatory T cells, and regulates experimental autoimmune arthritis. Clin Immunol 127: 78-88. doi: 10.1016/j.clim.2008.01.006 PMID: 18282810

67. Wang F, He W, Zhou H, Yuan J, Wu K, et al. (2007) The Tim-3 ligand galectin-9 negatively regulates CD8+ alloreactive T cell and prolongs survival of skin graft. Cell Immunol 250: 68-74. doi: 10.1016/j. cellimm.2008.01.006 PMID: 18353298

68. Jones RB, O'Connor R, Mueller S, Foley M, Szeto GL, et al. (2014) Histone deacetylase inhibitors impair the elimination of HIV-infected cells by cytotoxic T-lymphocytes. PLoS Pathog 10: e1004287. doi: 10.1371/journal.ppat.1004287 PMID: 25122219

69. Wang C, Abdel-Mohsen M, Strain MC, Lada SM, Yukl S, et al. (2014) Decreased HIV type 1 transcription in CCR5-Delta32 heterozygotes during suppressive antiretroviral therapy. J Infect Dis 210: 18381843. doi: 10.1093/infdis/jiu338 PMID: 24935955

70. Nishi N, Abe A, Iwaki J, Yoshida H, Itoh A, et al. (2008) Functional and structural bases of a cysteineless mutant as a long-lasting substitute for galectin-1. Glycobiology 18: 1065-1073. doi: 10.1093/ glycob/cwn089 PMID: 18796645

71. Nishi N, Itoh A, Fujiyama A, Yoshida N, Araya S, et al. (2005) Development of highly stable galectins: truncation of the linker peptide confers protease-resistance on tandem-repeat type galectins. FEBS Lett 579: 2058-2064. PMID: 15811318

72. Trapnell C, Roberts A, Goff L, Pertea G, Kim D, et al. (2012) Differential gene and transcript expression analysis of RNA-seq experiments with TopHat and Cufflinks. Nat Protoc 7: 562-578. doi: 10.1038/ nprot.2012.016 PMID: 22383036

73. Vandesompele J, De Preter K, Pattyn F, Poppe B, Van Roy N, et al. (2002) Accurate normalization of real-time quantitative RT-PCR data by geometric averaging of multiple internal control genes. Genome Biol 3: RESEARCH0034.

74. Stopak K, de Noronha C, Yonemoto W, Greene WC (2003) HIV-1 Vif blocks the antiviral activity of APOBEC3G by impairing both its translation and intracellular stability. Mol Cell 12: 591-601. PMID: 14527406

75. Gorny MK, Gianakakos V, Sharpe S, Zolla-Pazner S (1989) Generation of human monoclonal antibodies to human immunodeficiency virus. Proc Natl Acad Sci U S A 86: 1624-1628. PMID: 2922401

76. Fouchier RA, Simon JH, Jaffe AB, Malim MH (1996) Human immunodeficiency virus type 1 Vif does not influence expression or virion incorporation of gag-, pol-, and env-encoded proteins. J Virol 70: 82638269. PMID: 8970945

77. Simon JH, Fouchier RA, Southerling TE, Guerra CB, Grant CK, et al. (1997) The Vif and Gag proteins of human immunodeficiency virus type 1 colocalize in infected human T cells. J Virol 71:5259-5267. PMID: 9188594

78. Simon JH, Southerling TE, Peterson JC, Meyer BE, Malim MH (1995) Complementation of vif-defective human immunodeficiency virus type 1 by primate, but not nonprimate, lentivirus vif genes. $J$ Virol 69 : 4166-4172. PMID: 7769676

79. Vandergeeten C, Fromentin R, Merlini E, Lawani MB, DaFonseca S, et al. (2014) Cross-clade ultrasensitive PCR-based assays to measure HIV persistence in large-cohort studies. J Virol 88: 1238512396. doi: 10.1128/JVI.00609-14 PMID: 25122785

80. Benjamini Y, Drai D, Elmer G, Kafkafi N, Golani I (2001) Controlling the false discovery rate in behavior genetics research. Behav Brain Res 125: 279-284. PMID: 11682119 